\title{
Neonatal Fc receptor antagonist efgartigimod safely and sustainably reduces IgGs in humans
}

\author{
Peter Ulrichts, ${ }^{1}$ Antonio Guglietta, ${ }^{1}$ Torsten Dreier, ${ }^{1}$ Tonke van Bragt, ${ }^{1}$ Valérie Hanssens, ${ }^{1}$ Erik Hofman, ${ }^{1}$ Bernhardt Vankerckhoven, ${ }^{1}$ \\ Peter Verheesen, ${ }^{1}$ Nicolas Ongenae, ${ }^{1}$ Valentina Lykhopiy, ${ }^{1}$ F. Javier Enriquez, ${ }^{1}$ JunHaeng Cho, ${ }^{2}$ Raimund J. Ober, ${ }^{2,3}$ E. Sally Ward, ${ }^{2,4}$ \\ Hans de Haard, ${ }^{1}$ and Nicolas Leupin ${ }^{1}$
}

'argenx BVBA, Zwijnaarde, Belgium. ²Department of Molecular and Cellular Medicine, Texas A\&M University Health Science Center, College Station, Texas, USA. ${ }^{3}$ Department of Biomedical Engineering, Texas A\&M University, College Station, Texas, USA. ${ }^{4}$ Department of Microbial Pathogenesis and Immunology, Texas A\&M University Health Science Center, Bryan, Texas, USA

BACKGROUND. Intravenous Ig (IVIg), plasma exchange, and immunoadsorption are frequently used in the management of severe autoimmune diseases mediated by pathogenic IgG autoantibodies. These approaches modulating IgG levels can, however, be associated with some severe adverse reactions and a substantial burden to patients. Targeting the neonatal Fc receptor (FcRn) presents an innovative and potentially more effective, safer, and more convenient alternative for clearing pathogenic IgGs.

METHODS. A randomized, double-blind, placebo-controlled first-in-human study was conducted in 62 healthy volunteers to explore single and multiple ascending intravenous doses of the FcRn antagonist efgartigimod. The study objectives were to assess safety, tolerability, pharmacokinetics, pharmacodynamics, and immunogenicity. The findings of this study were compared with the pharmacodynamics profile elicited by efgartigimod in cynomolgus monkeys.

RESULTS. Efgartigimod treatment resulted in a rapid and specific clearance of serum IgC levels in both cynomolgus monkeys and healthy volunteers. In humans, single administration of efgartigimod reduced IgC levels up to $50 \%$, while multiple dosing further lowered IgGs on average by $75 \%$ of baseline levels. Approximately 8 weeks following the last administration, IgG levels returned to baseline. Efgartigimod did not alter the homeostasis of albumin or Igs other than IgG, and no serious adverse events related to efgartigimod infusion were observed.

CONCLUSION. Antagonizing FcRn using efgartigimod is safe and results in a specific, profound, and sustained reduction of serum IgC levels. These results warrant further evaluation of this therapeutic approach in IgG-driven autoimmune diseases.

TRIAL REGISTRATION. Clinicaltrials.gov NCT03457649.

FUNDING. argenx BVBA.

\section{Introduction}

Autoimmune diseases originate from a failure of the immune system to discriminate between self and nonself antigens. As a result, the immune system attacks normal constituents of the body, which may lead to tissue damage. A wide variety of autoimmune disorders have been described, affecting $7.6 \%$ to $9.4 \%$ of the global population (1), and this group of diseases involves almost every single system in the body (2). The mainstay therapy consists of corticosteroids that can be combined with other immunosuppressive agents, which are hampered by a small therapeutic window to balance efficacy with unwanted side effects. For several autoimmune disorders, includ-

Role of funding source: argenx BVBA was the funder of this study and formal sponsor of the clinical trial.

Conflict of interest: PU, AG, TD, VH, EH, BV, PV, NO, VL, FJE, HDH, and NL are or used to be full-time employees of argenx BVBA. TVB is a consultant to argenx BVBA. ESW, RJO, and JC are supported in part by a research grant funded by argenx BVBA

Submitted: November 13, 2017; Accepted: July 3, 2018.

Reference information: J Clin Invest. 2018;128(10):4372-4386.

https://doi.org/10.1172/JCI97911. ing multiple sclerosis, myasthenia gravis, pemphigus vulgaris, primary immune thrombocytopenia, and systemic lupus erythematosus, autoreactive IgG Abs play a central role in disease pathology, and decreasing autoantibody levels from the periphery or B cell depletion are effective therapeutic strategies (3). Such IgG-reducing therapies include plasmapheresis, i.v. Ig (IVIg), and immunoadsorption, which all give satisfactory clinical responses in a wide variety of IgG-driven autoimmune indications $(4,5)$. However, because of the high costs and side effects of these therapies, the development of alternative pathogenic IgG-reducing strategies is of great interest.

One such strategy involves inhibition of the neonatal Fc receptor (FcRn) (6). Originally, this receptor was thought to transfer maternal IgGs to neonates in rodents (7), but more recently, FcRn has been shown to also play a central role in trafficking IgGs and albumin into recycling pathways $(8,9)$. Briefly, following the uptake of IgGs into FcRn-expressing cells through pinocytosis, their Fc regions bind to FcRn in early acidified endosomes $(\mathrm{pH}<6.5)$ with high affinity. In complex with FcRn, IgGs are subsequently recycled or transcytosed to the cell surface and released from the complex at the near-neutral $\mathrm{pH}$ (pH 7.4) of the extracellular space $(10,11)$. This pH-dependent sal- 


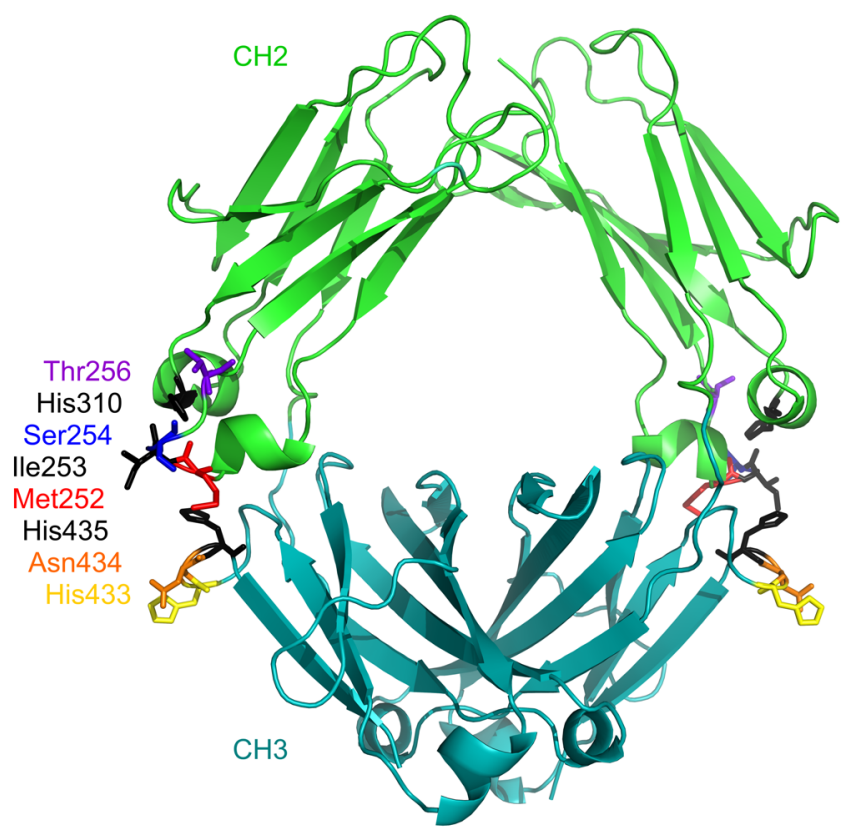

Figure 1. The location of the residues targeted for mutation in efgartigimod. X-ray crystallographic structure of hlgG1-derived Fc (54) with locations of residues that are mutated in efgartigimod shown in color (Met252 to Tyr, Ser254 to Thr, Thr256 to Glu, His433 to Lys, and Asn434 to Phe). Residues playing a central role in hlgG1:FcRn interaction are indicated in black (Ile253, His310, and His435) (55). The figure was generated using PyMOL (PyMOL Molecular Graphics System, version 2.0, Schrödinger LLC).

vage pathway is accountable for the relatively high concentration of IgG in the circulation and for the long half-life of IgG compared with other Ig isotypes, such as IgA and IgM, which do not undergo recycling $(12,13)$. Therefore, molecules that block the interaction of FcRn with IgGs are expected to induce degradation and fast clearance of IgGs. Albumin is also recycled by FcRn in a similar pH-dependent fashion, but it interacts at a site distinct from that of IgG, and therefore, binding of albumin and IgG can occur concurrently (14).

Efgartigimod is a human IgG1-derived (hIgG1-derived) Fc fragment that has been modified using ABDEG technology to increase its affinity for FcRn at both physiological and acidic $\mathrm{pH}$, with retention of $\mathrm{pH}$ dependence (higher affinity at $\mathrm{pH} 6.0$ than at near-neutral $\mathrm{pH}$ ) characteristic of FcRn interactions (15). Molecules encompassing these ABDEG mutations (M252Y/S254T/ T256E/H433K/N434F) have been shown to block FcRn function, and injection of an $\mathrm{ABDEG}$-equipped $\mathrm{Ab}$ in mice resulted in a very rapid lowering of endogenous IgG levels (15). The therapeutic applicability of this concept was demonstrated in various murine models of IgG-mediated autoimmunity $(16,17)$.

Here, the FcRn-antagonizing properties of efgartigimod in cynomolgus monkeys are described as well as the results of a firstin-human study evaluating the pharmacokinetic (PK), pharmacodynamic (PD), and safety profile of efgartigimod in healthy volunteers.

\section{Results}

In vitro characterization of efgartigimod. Efgartigimod is a hIgG1derived Fc fragment (residues D220-K447) from the za allotype that has been mutated at 5 residues, the so-called ABDEG muta- tions (15), to increase its affinity for FcRn at both acidic and neutral $\mathrm{pH}$ (Figure 1). SPR analyses demonstrate that efgartigimod has equilibrium dissociation constants for human FcRn of 14.2 $\mathrm{nM}$ and $320 \mathrm{nM}$ at pH 6.0 and pH 7.4, respectively (Supplemental Figure 1; supplemental material available online with this article; https://doi.org/10.1172/JCI97911DS1). These affinities are analogous to those of a full-length IgG (anti-hen egg lysozyme [antiHEL]) with the ABDEG mutations (ref. 15 and data not shown).

In vitro studies with transfected endothelial cells (HMEC-1) expressing human FcRn-GFP $(10,11)$ were performed using both flow cytometry and fluorescence microscopy. Quantitation of the accumulation of fluorescently labeled efgartigimod (pulsed at $25 \mu \mathrm{g} / \mathrm{ml}$ at $\mathrm{pH}$ 7.4) by flow cytometry immediately following a pulse of the transfected cells resulted in higher levels of cellassociated efgartigimod than WT hIgG1 (HEL specific; pulsed at $200 \mu \mathrm{g} / \mathrm{ml}$ ), consistent with the increase in affinity of efgartigimod for human FcRn at near-neutral pH (Figure 2, A and B, and Supplemental Table 1). Following a chase period of 30 minutes, $39.2 \% \pm 3.4 \%$ and $22.5 \% \pm 3.6 \%$ of fluorescently labeled efgartigimod and hIgG1, respectively, remained within the cells. The data for hIgG1 are consistent with our earlier studies demonstrating that this $\mathrm{Ab}$ is efficiently recycled from FcRn-expressing cells to almost background levels within 30 minutes (Figure 2B and Supplemental Table 1). Although efgartigimod is also recycled from the cells, the relatively high levels of accumulation of efgartigimod within cells during the pulse phase resulted in substantially greater cell-associated levels compared with that in hIgG1 following the 120-minute chase period (Figure 2A and Supplemental Table 1). As a comparator, we also analyzed the behavior of an $\mathrm{Ab}$ that binds with dissociation constants of $3.9 \mathrm{nM}$ and 6.2 $\mathrm{nM}$ to human FcRn at $\mathrm{pH} 6.0$ and pH 7.4, respectively, through its Fab arms (18) (Supplemental Figure 2). This anti-FcRn Ab accumulated to higher levels than efgartigimod within cells during a 30-minute pulse and remained at similar levels during the 2-hour chase period (Figure 2, A and B).

In addition to the flow cytometry experiments, comparison of the behavior of efgartigimod and the anti-FcRn Ab using fluorescence microscopy demonstrated that both $\mathrm{FcRn}$ ligands were extensively colocalized with $\mathrm{FcRn}$ within subcellular compartments following a 30-minute pulse (Figure 2C). Our earlier studies analyzing the subcellular localization of FcRn-GFP in transfected HMEC-1 cells have demonstrated that these intracellular compartments are early/late endosomes and tubulovesicular transport carriers (recycling compartments) $(11,19,20)$. Following 16 hours of chase, the anti-FcRn Ab was present in dextranpositive lysosomes (Figure 2C). Under the same conditions of imaging, substantially lower levels of Alexa Fluor 647-labeled efgartigimod were observed within cells following a 16-hour chase period, although linear adjustment of the Alexa Fluor 647 intensity also revealed colocalization of this $\mathrm{Fc}$ fragment with FcRn-GFP-positive compartments and dextran-positive lysosomes following this chase time (Figure 2C and Supplemental Figure 3). Collectively, the flow cytometry and microscopy data indicate that the higher affinity and/or avidity of the anti-FcRn $\mathrm{Ab}$ relative to efgartigimod confers greater retention in FcRnpositive compartments within cells, combined with increased lysosomal accumulation. 
A

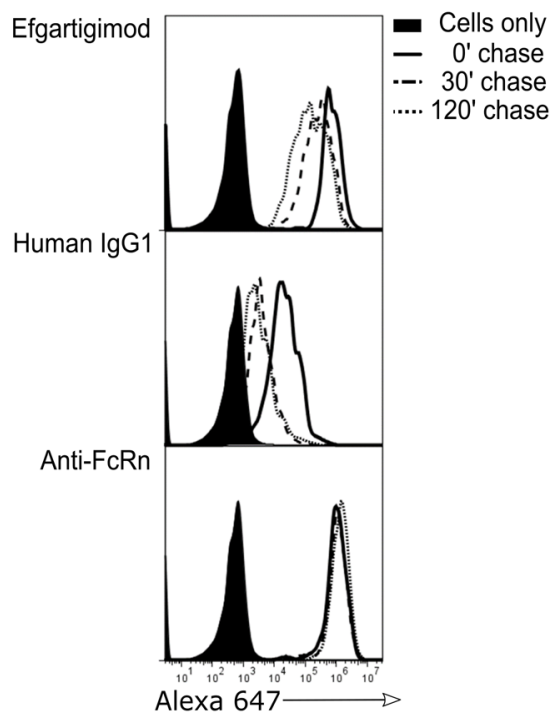

B

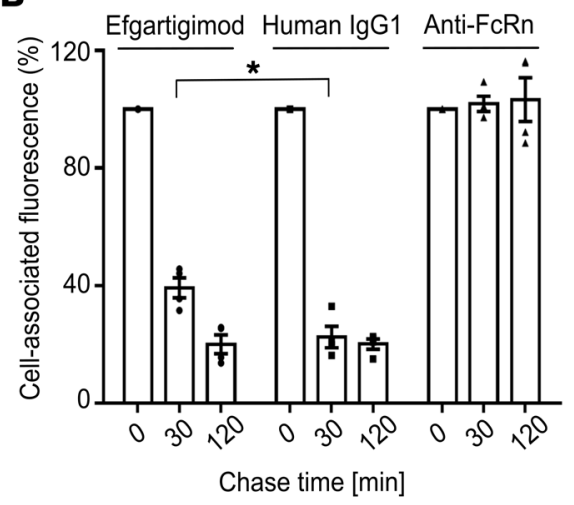

C
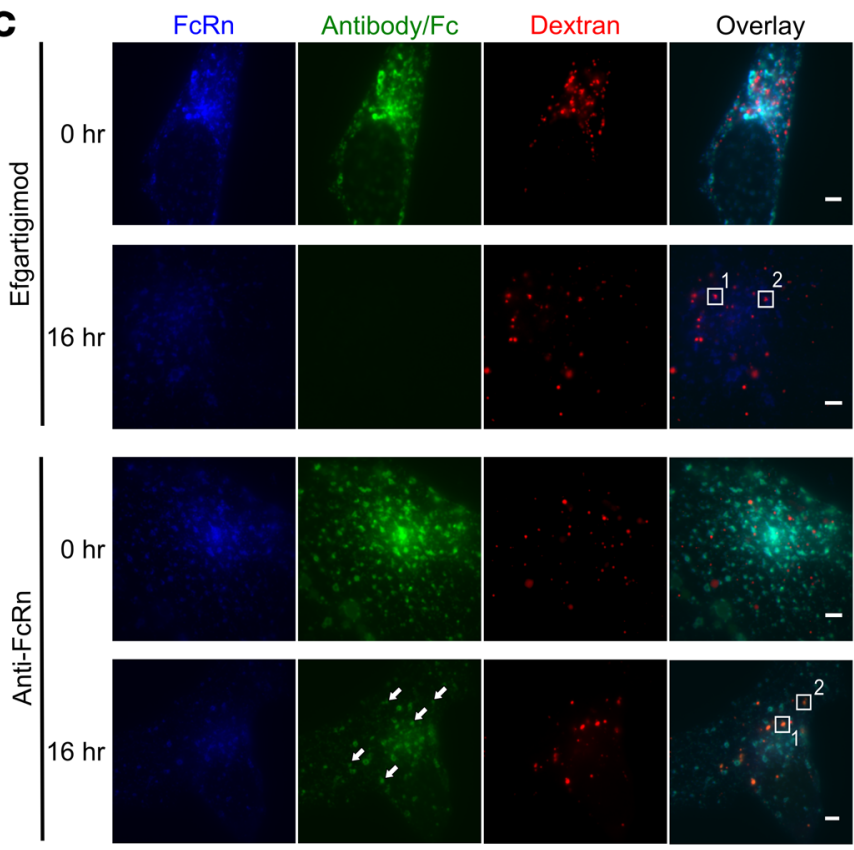

Efgartigimod

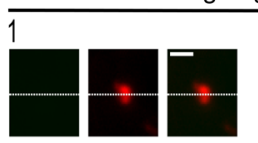

-Efgartigimod

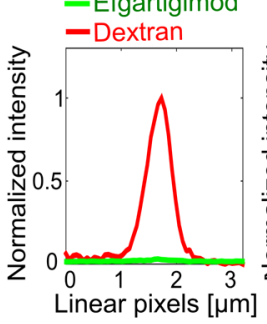

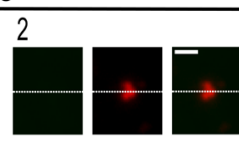

- Efgartigimod

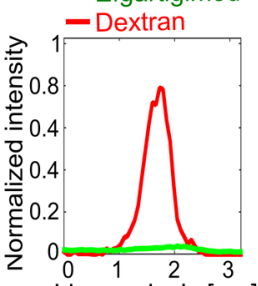

Linear pixels $[\mu \mathrm{m}]$

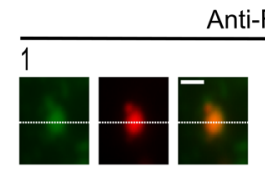

- Anti-FcRn

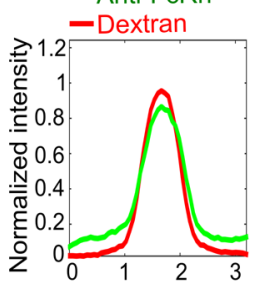

Linear pixels $[\mu \mathrm{m}]$

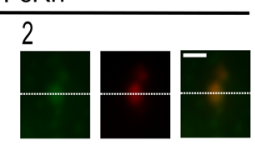

- Anti-FcRn

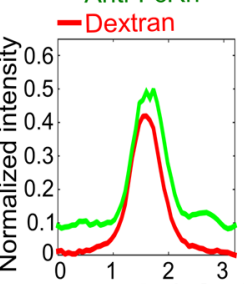

Linear pixels [ $\mu \mathrm{m}]$

Figure 2. Differences in subcellular trafficking behavior between efgartigimod and anti-FcRn Ab. (A) HMEC-1 cells expressing hFcRn-GFP were pulsechased with Alexa Fluor 647-labeled efgartigimod, isotype control (HEL-specific hlgG1), or anti-FcRn Ab at concentrations of 25, 200, and 75 $\mu \mathrm{g} / \mathrm{ml}$, respectively, for 30 minutes and chased for 0, 30, and 120 minutes. Levels of cell-associated Alexa Fluor 647 were determined using flow cytometry. Representative histogram plots for 2 independent experiments are shown. (B) Cell-associated fluorescence levels for analyses carried out as in panel A, displayed as the percentage of fluorescence remaining at different chase times relative to the pulse-only samples. For chase times of 30 and 120 minutes, means of 4 samples (2 samples per experiment, 2 experiments) are shown. Error bars indicate SEM, and statistical significance of the difference between efgartigimod and hlgG1 following a 30-minute chase is indicated. ${ }^{*} P<0.05$, 1-way ANOVA. (C) HMEC-1 cells expressing human FcRn-GFP were pulse-chased with $500 \mu \mathrm{g} / \mathrm{ml}$ Alexa Fluor 555-labeled dextran and then pulse-chased (30-minute pulse, 0- or 16-hour chase) with Alexa Fluor 647-labeled efgartigimod (25 $\mu \mathrm{g} / \mathrm{ml})$ or anti-FcRn Ab $(75 \mu \mathrm{g} / \mathrm{ml})$. Images of representative cells are shown, with arrowheads in the anti-FcRn panel (16 hours) indicating Ab that is localized in dextran-positive compartments. Data for Alexa Fluor 647-labeled efgartigimod or Alexa Fluor 647-labeled anti-FcRn and FcRn-GFP are displayed as acquired. The lysosomes in boxed regions (labeled 1 and 2 for each inhibitor) are cropped and expanded (lower panels), and pixel intensities along the dotted lines are shown. Intensities are normalized against the highest pixel value in each 16-hour image (Alexa Fluor 555) or for both 16-hour images (Alexa Fluor 647). GFP, Alexa Fluor 555, and Alexa Fluor 647 are pseudocolored blue, red, and green, respectively. Scale bars: $3 \mu \mathrm{m}$ (upper panels); $1 \mu \mathrm{m}$ (cropped lysosomes). Microscopy data are representative of at least 2 independent experiments.

Effect of efgartigimod and IVIg on tracer clearance in cynomolgus monkeys. Since the FcRn-binding properties of cynomolgus monkey IgG and human IgG are very similar (21) and efgartigimod showed an increase in affinity to human FcRn similar to that shown with cynomolgus FcRn (Supplemental Figure 4), cynomolgus monkey was considered a relevant species to explore the PD properties of efgartigimod.

To test the effect of efgartigimod on IgG homeostasis in cynomolgus monkeys, animals were injected with a non-target-binding hIgG1 tracer Ab (FR70-hIgG1, targeting mouse CD70, not cross- reactive to human or monkey target), followed 2 days later with an i.v. infusion of $70 \mathrm{mg} / \mathrm{kg}$ efgartigimod. An infusion with $2 \mathrm{~g} / \mathrm{kg}$ IVIg was included as control, as this is a standard therapy in many autoimmune indications and is known to accelerate $\operatorname{IgG}$ clearance (22). A single infusion of $70 \mathrm{mg} / \mathrm{kg}$ efgartigimod to monkey cleared the human FR70-hIgG1 tracer Ab levels more rapidly and more efficiently $(>95 \%$ clearance in 4 days, no tracer detected afterwards) than IVIg at a dose used in clinical settings ( $75 \%$ clearance in 7 days) (Figure 3). These data confirm earlier data in rodents (15) and indicate that inhibition of FcRn function by 


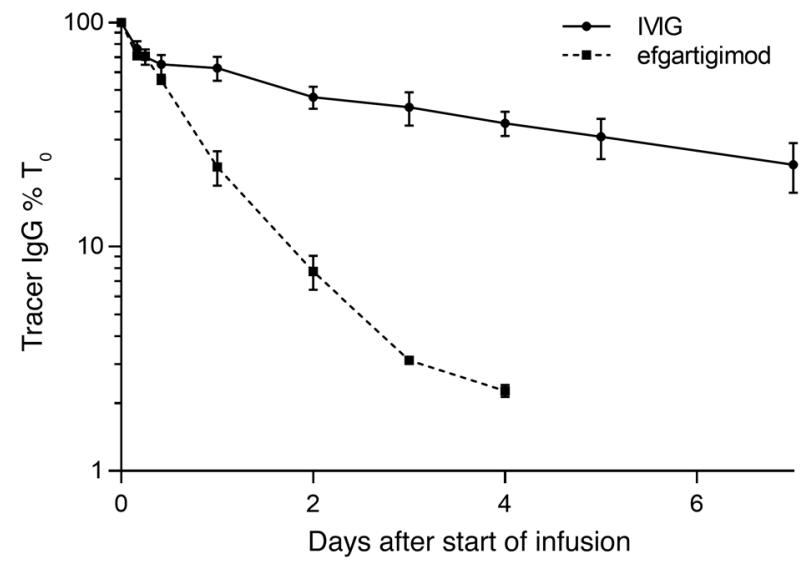

Figure 3. Effect of efgartigimod and IVIg on tracer lgG levels in cynomolgus monkeys. Animals ( $n=2 /$ group) were injected with $1 \mathrm{mg} / \mathrm{kg}$ of the tracer Ab FR70-hlgG1, followed 2 days later with i.v. infusion of $70 \mathrm{mg} /$ $\mathrm{kg}$ efgartigimod or $2 \mathrm{~g} / \mathrm{kg}$ IVIg. Tracer levels in serum, detected by murine CD70-binding ELISA, were plotted relative to predose levels. Values are shown as mean $\pm \mathrm{SEM}$. $\% \mathrm{~T}_{0}$, percentage change from baseline.

efgartigimod is driven by its competitive binding advantage over endogenous IgGs and not, as with IVIg, by saturation of the FcRn receptor due to a massive increase in IgG levels.

Dose-escalation study in cynomolgus monkey. In a subsequent study using an experimental setup identical to that described above, doses of efgartigimod ranging from 0.2 to $200 \mathrm{mg} / \mathrm{kg}$ were infused and both tracer $\operatorname{IgG}$ and endogenous cynomolgus monkey IgG levels were determined (Figure 4, A and B). Neither the human FR70-hIgG1 tracer Ab nor endogenous IgG levels were affected by a single dose of efgartigimod at $0.2 \mathrm{mg} / \mathrm{kg}$ compared with vehicle, but enhanced clearance of both tracer and endogenous IgG was observed for cynomolgus monkeys dosed at 2, 20, or $200 \mathrm{mg} / \mathrm{kg}$ (data from the $70 \mathrm{mg} / \mathrm{kg}$ efgartigimod group from the previous experiment were included as reference). A maximal reduction of 55\% in endogenous IgG levels was observed following a single administration of efgartigimod, and this PD effect leveled out at doses starting from $20 \mathrm{mg} / \mathrm{kg}$. Based on the latter finding and the increasing presence of efgartigimod in the urine at doses from $20 \mathrm{mg} / \mathrm{kg}$ and above (data not shown), the saturating dose was considered to be close to $20 \mathrm{mg} / \mathrm{kg}$. An average half-life of 38 hours was calculated for efgartigimod at this dose (data not shown). Efgartigimod specifically influenced IgG catabolism, as no effect was seen on IgA and IgM levels (Supplemental Figure 5).

Multiple dose study in cynomolgus monkey. The hypothesis that repeated infusions of efgartigimod may lower IgG levels to a greater extent than a single infusion was next investigated. Two strategies were followed: one group of monkeys received an efgartigimod infusion every 24 hours during the first 4 days,

Figure 4. Efgartigimod dose-escalation study in cynomolgus monkeys. Animals ( $n=2$ /group) were injected with $1 \mathrm{mg} / \mathrm{kg}$ of the tracer Ab FR70hlgG1, followed 2 days later with i.v. infusion of 0.2 to $200 \mathrm{mg} / \mathrm{kg}$ efgartigimod or vehicle. Tracer levels in serum (A) are detected by murine CD70binding ELISA. Endogenous IgG levels in serum (B) were plotted relative to predose levels. Cyno, cynomolgus. Values are shown as mean \pm SEM. while the other group received the drug once every 4 days. Efgartigimod was administered at $20 \mathrm{mg} / \mathrm{kg}$. A clear difference between the 2 groups was observed from day 7 onwards (Figure 5). Dosing efgartigimod once every 4 days resulted in a more profound and longer reduction of IgG levels than dosing every 24 hours for 4 days. A maximum reduction in IgG levels of $75 \%$ was observed after multiple dosing.

Phase I study in healthy volunteers. A randomized, doubleblind, placebo-controlled first-in-human study was initiated to explore i.v. single ascending doses (SAD) and multiple ascending doses (MAD) of efgartigimod. The objectives of this study were to assess the safety, tolerability, PK, PD, and immunogenicity of efgartigimod in healthy males and healthy females of nonchildbearing potential. Subjects in the SAD part (cohorts 1-5) were dosed either with placebo or $0.2,2,10,25$, or $50 \mathrm{mg} /$ $\mathrm{kg}$ efgartigimod (Figure 6). Three different dosing regimens were explored in the MAD phase. Volunteers were either dosed with $10 \mathrm{mg} / \mathrm{kg}$ efgartigimod or placebo every 4 days (q4d) on 6 occasions, $10 \mathrm{mg} / \mathrm{kg}$ efgartigimod or placebo every 7 days (q7d) on 4 occasions, or $25 \mathrm{mg} / \mathrm{kg}$ efgartigimod or placebo q7d on 4 occasions (Figure 7).

PK of efgartigimod in healthy volunteers. Serum levels of efgartigimod were measured in both the SAD and MAD part of this study. Measurements were performed using an efgartigimodspecific ELISA, and PK parameters were calculated using a noncompartmental method.
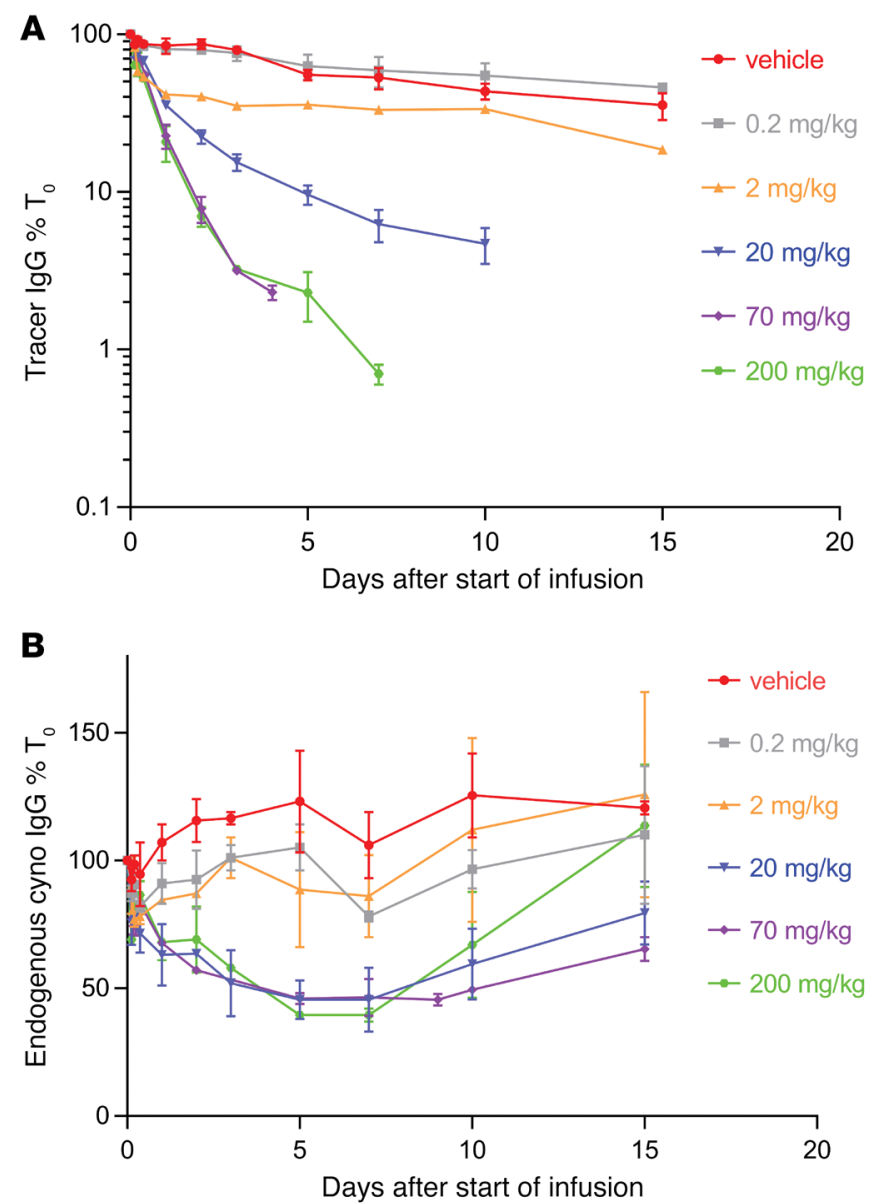


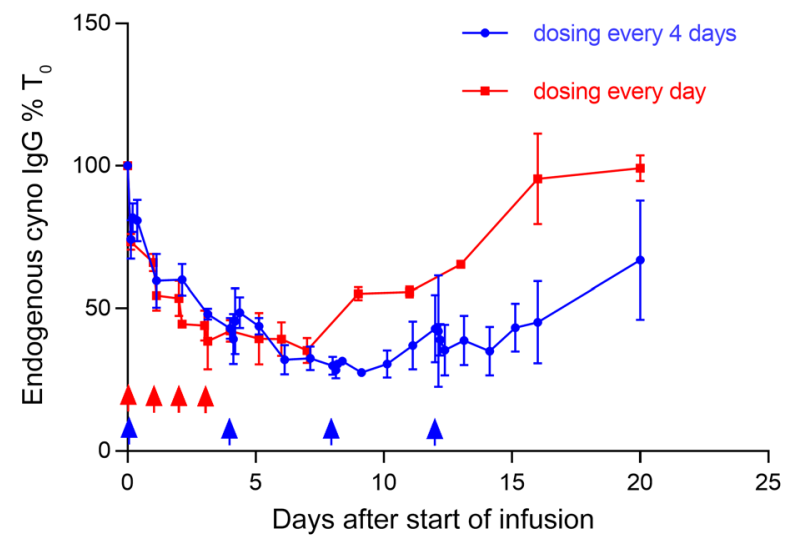

In the $\mathrm{SAD}$ phase, the mean $\mathrm{C}_{\max }$ increased more than dose proportionally for the 0.2 and $2 \mathrm{mg} / \mathrm{kg}$ cohorts and then overall dose proportionally between 10 and $50 \mathrm{mg} / \mathrm{kg}$, up to a $\mathrm{C}_{\max }$ value of $1,175 \mu \mathrm{g} / \mathrm{ml}$ (Figure 8 and Table 1). Area under the serum concentration time curve from time zero to infinity $\left(\mathrm{AUC}_{\mathrm{inf}}\right)$ values increased overall dose proportionally between 2 and 50 $\mathrm{mg} / \mathrm{kg}$. In all dose groups, the median time to reach $\mathrm{C}_{\max }$ was 2.0 hours (i.e., the end of the infusion). The half-life of efgartigimod was determined as between 85.1 and 104 hours over 2 to $50 \mathrm{mg} / \mathrm{kg}$ and as 140 hours at $0.2 \mathrm{mg} / \mathrm{kg}$. The mean clearance (CL) values ranged from 0.122 to $0.163 \mathrm{l} / \mathrm{h}$ and mean volume of distribution $\left(\mathrm{V}_{\mathrm{z}}\right)$ values ranged from 14.8 to $21.4 \mathrm{l}$, which is at the high end of what is typically observed for biologics (23), indicating that efgartigimod is efficiently distributed from the circulation into the tissues. Additionally, the presence of efgartigimod in the urine was studied up to 72 hours after infusion. For the 0.2 and $2.0 \mathrm{mg} / \mathrm{kg}$ treatment groups, efgartigimod was
Figure 5. Endogenous IgC levels in multiple dose study in cynomolgus monkeys. Animals ( $n=2 /$ group) were injected i.v. with $20 \mathrm{mg} / \mathrm{kg}$ efgartigimod every day during the first 4 days or once every 4 days (arrows indicate drug administration). Endogenous IgG levels in serum were plotted relative to predose levels. Values are shown as mean \pm SEM.

not quantifiable in urine over the 0 - to 72 -hour period. The mean total amounts excreted in urine over the 0 - to 72 -hour period were minimal, with values of $0.100 \mathrm{mg}(0.0124 \%$ of the dose), $0.474 \mathrm{mg}(0.0238 \%$ of the dose $)$, and $3.39 \mathrm{mg}(0.0845 \%$ of the dose) for the 10,25 , and $50 \mathrm{mg} / \mathrm{kg}$ doses, respectively. The excretion in urine was rapid as $100 \%$, about $82 \%$, and about $55 \%$ of the amount over 72 hours was excreted within the first 12 hours for the 10, 25, and $50 \mathrm{mg} / \mathrm{kg}$ doses, respectively.

In the MAD part of the study, the efgartigimod PK parameters after the first administration in all treatment groups were consistent with those observed in the SAD part of the study (Figure 9).

From the first to the last dose, efgartigimod $\mathrm{C}_{\max }$ reached values of $161-229 \mu \mathrm{g} / \mathrm{ml}(10 \mathrm{mg} / \mathrm{kg} \mathrm{q} 4 \mathrm{~d}), 195-237 \mu \mathrm{g} / \mathrm{ml}(10 \mathrm{mg} / \mathrm{kg}$ q7d), and 393-535 $\mu \mathrm{g} / \mathrm{ml}$ (25 mg/kg q7d) (Table 2). The elimination phase was parallel and multiphasic for all doses and after all administrations. Efgartigimod concentrations were still quantifiable in serum of all subjects 21 days after the last dose. Overall, no accumulation of efgartigimod was observed after dosing q4d for 21 days and dosing $\mathrm{q} 7 \mathrm{~d}$ for 22 days, with mean accumulation ratio $\left(\mathrm{R}_{\mathrm{ac}}\right)$ values ranging from 0.814 to 1.26 . The PK profiles after the last dosing day (i.e., after multiple administrations) were similar to those after the first dose.

$P D$ of efgartigimod in healthy volunteers. A detailed PD analysis was performed in both parts of the study, thereby monitoring the levels of the individual IgG subclasses (IgG1 through IgG4).

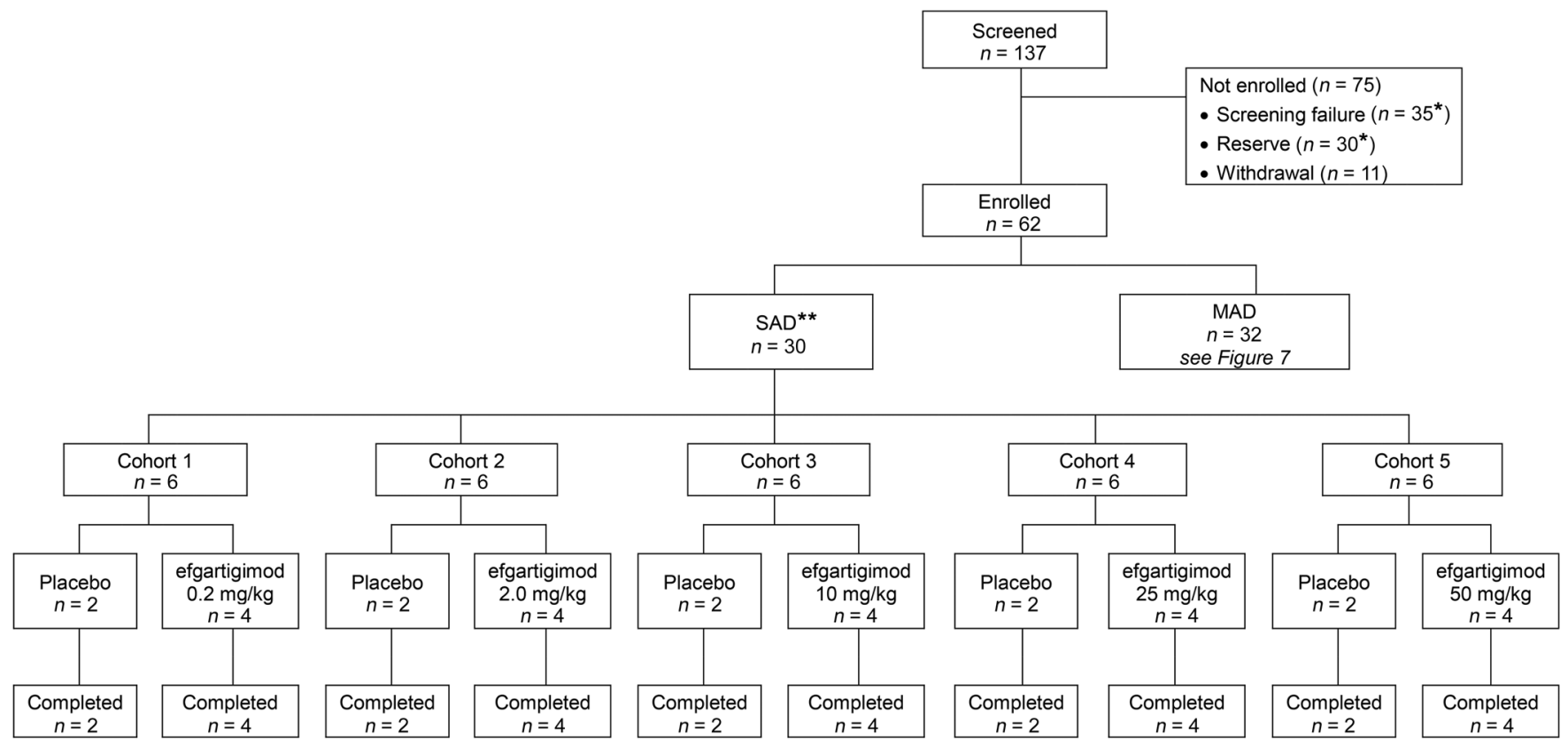

Figure 6. Flow chart of SAD part of first-in-human study of efgartigimod in healthy volunteers. $n$, number of subjects. ${ }^{*}$ One subject who did not originally fulfill the in- and exclusion criteria was rescreened at a later stage and was subsequently listed as a reserve subject. ${ }^{* *}$ An optional sixth cohort was not initiated. 


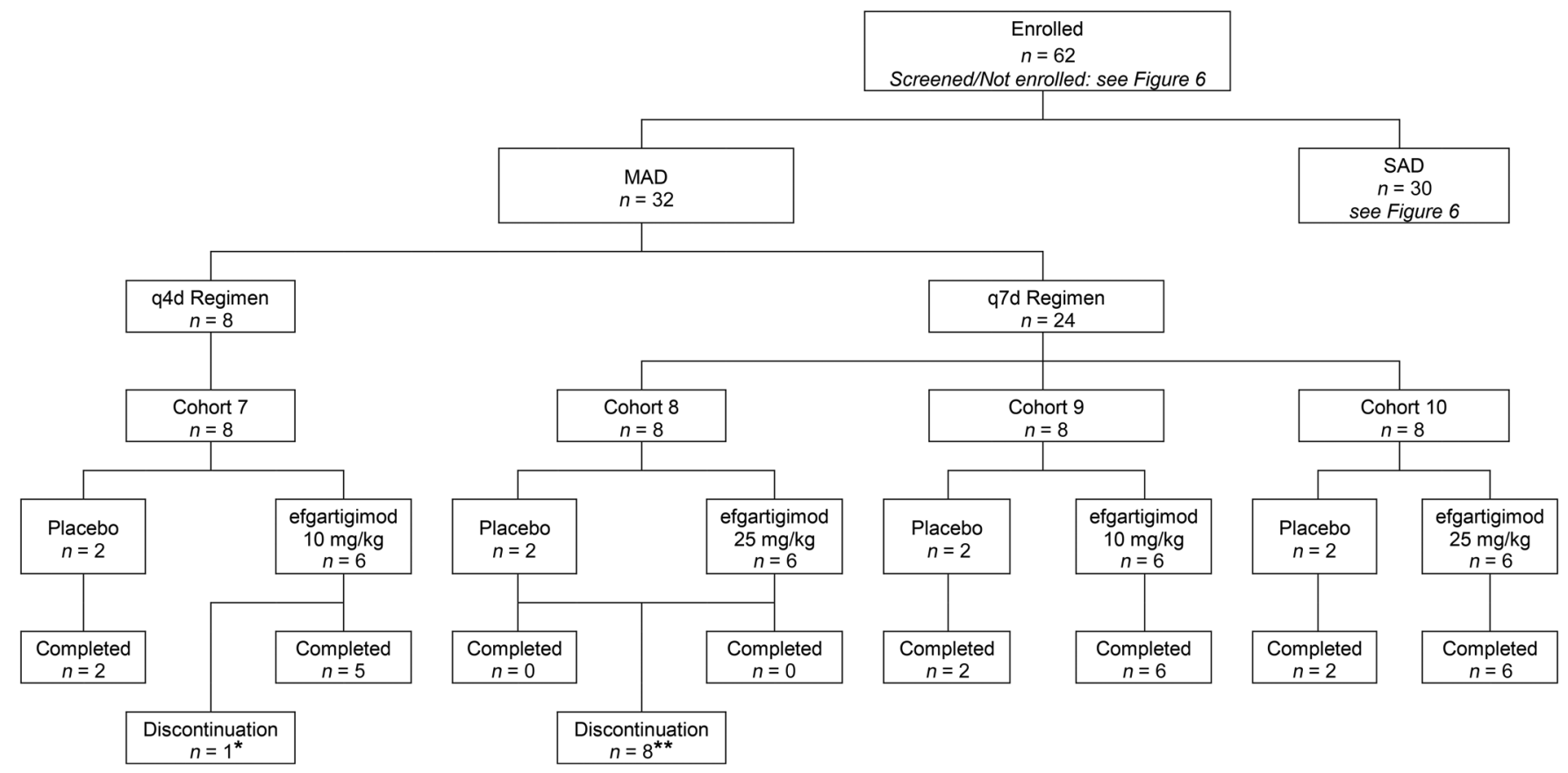

Figure 7. Flow chart of MAD part of first-in-human study of efgartigimod in healthy volunteers. $n$, number of subjects. ${ }^{*}$ Subject withdrew consent. ${ }^{*}$ Dosing of all subjects in cohort 8 was discontinued early because of 1 subject experiencing a SAE. At that time, 4 subjects in this cohort had received 2 doses of the study drug and 4 subjects, including the subject with the SAE, had received 1 dose. After further investigation, it was considered unlikely that the SAE was related to the study drug.

A rapid, sustained, and dose-dependent reduction of IgG levels was observed after administration of a single dose of 2 to $50 \mathrm{mg} / \mathrm{kg}$ efgartigimod (SAD part) compared with placebo (Figure 10).

The IgG1 levels decreased from 2 days after delivery of the 2 $\mathrm{mg} / \mathrm{kg}$ dose and had already decreased from 1 day at 10, 25, and $50 \mathrm{mg} / \mathrm{kg}$. This decrease was at its maximum at 6 to 21 days after delivery for the $2,10,25$, and $50 \mathrm{mg} / \mathrm{kg}$ doses and was sustained over time with $34.4 \%, 36.5 \%$, and $42.2 \%$ reductions of IgG1 levels, compared with baseline, at 28 days after delivery (the last sample collection time) for the 10,25 , and $50 \mathrm{mg} / \mathrm{kg}$ doses, respectively. These findings were confirmed by the percentage changes of maximum percentage reduction value $\left(\mathrm{E}_{\max }\right)$ and area under the percentage of reduction curve (AUEC) (Supplemental Table 2), which were superior for the 10 to $50 \mathrm{mg} / \mathrm{kg}$ doses as compared with the $0.2 \mathrm{mg} / \mathrm{kg}$ and $2 \mathrm{mg} / \mathrm{kg}$ doses. No significant differences within the 10 to $50 \mathrm{mg} / \mathrm{kg}$ dose range were seen in terms of $\mathrm{E}_{\max }$ or AUEC; thus, the maximum effect was already reached with the $10 \mathrm{mg} / \mathrm{kg}$ dose (Supplemental Table 2).

In the SAD part, the levels of IgG subclasses 2 and 3 were reduced in a way that was similar to the IgG1 levels over time, while IgG4 levels were reduced to a slightly lower extent. For the doses of 10 to $50 \mathrm{mg} / \mathrm{kg}$, the mean $\mathrm{E}_{\max }$ ranged from $49.1 \%$ to $58.3 \%$ for IgG1, from $42.4 \%$ to $52.3 \%$ for IgG2, and from $54.0 \%$ to $61.1 \%$ for IgG3, while it ranged from $36.6 \%$ to $46.0 \%$ for IgG4. The administration of single doses of efgartigimod $(0.2$ to $50 \mathrm{mg} /$ $\mathrm{kg}$ ) did not induce a relevant decrease in IgA, IgD, IgE, or IgM or in albumin serum levels (data not shown).

After multiple administrations at $10 \mathrm{mg} / \mathrm{kg} \mathrm{q} 4 \mathrm{~d}$ and $\mathrm{q} 7 \mathrm{~d}$ and at $25 \mathrm{mg} / \mathrm{kg}$ q7d (MAD part), the reduction of IgG levels was enhanced compared with that seen with a single infusion of efgartigimod (Figure 11). The decrease of IgG1 levels compared with placebo was at its maximum after the last administration in all treatment groups. The respective mean $\mathrm{E}_{\max }$ (calculated over the whole administration period) ranged from $73 \%$ to $78.5 \%$ for the 3 dosing regimens (Supplemental Table 3). After the last administration, the IgG1 levels increased again to about $50 \%$ reduction at day 42 or day 43 for q $4 \mathrm{~d}$ or q7d regimens, respectively, and returned to around baseline levels by day 77 or day 78 for $\mathrm{q} 4 \mathrm{~d}$ or $\mathrm{q} 7 \mathrm{~d}$ regimens, respectively. These findings indicate a reversible drug effect. There was no significant difference in reduction of IgG1 levels between the q4d and

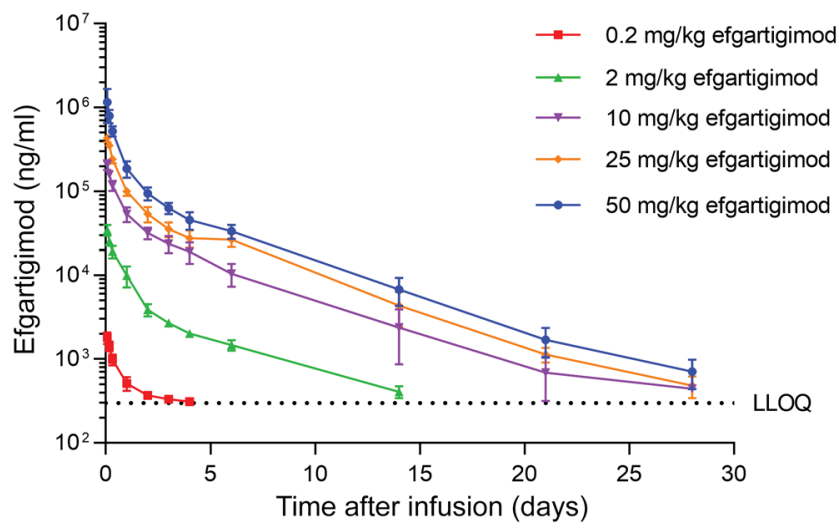

Figure 8. Efgartigimod serum concentration in the SAD part of first-inhuman study. Healthy subjects ( $n=6$ /group) were dosed with $0.2,2,10$, 25 , or $50 \mathrm{mg} / \mathrm{kg}$ efgartigimod or placebo (i.e., cohorts 1-5). Efgartigimod or placebo (randomized at a 4:2 ratio) was administered i.v. in a 2-hour infusion. Values are shown as mean \pm SD. LLOQ, lower limit of quantification. 


\section{Table 1. Summary of efgartigimod PK parameters for SAD part}

\begin{tabular}{|c|c|c|c|c|c|}
\hline PK parameter & $\begin{array}{c}0.2 \mathrm{mg} / \mathrm{kg} \\
n=4\end{array}$ & $\begin{array}{c}2.0 \mathrm{mg} / \mathrm{kg} \\
n=4\end{array}$ & $\begin{array}{c}10 \mathrm{mg} / \mathrm{kg} \\
n=4\end{array}$ & $\begin{array}{c}25 \mathrm{mg} / \mathrm{kg} \\
n=4\end{array}$ & $\begin{array}{c}50 \mathrm{mg} / \mathrm{kg} \\
n=4\end{array}$ \\
\hline $\operatorname{AUC}_{\text {inf }}(\mu \mathrm{g} . h / \mathrm{ml})$ & $N C^{n=0}$ & $998(5.89)$ & $6,818(22.3)$ & $12,826(16.4)$ & $23,435(13.0)$ \\
\hline$t_{1 / 2, \lambda}(h)$ & $140(78.2)^{n=3}$ & $104(7.55)$ & $85.1(8.81)$ & $89.7(2.60)$ & $91.3(5.31)$ \\
\hline CL (I/h) & $N C^{n}=0$ & $0.142(13.8)$ & $0.122(22.0)$ & $0.153(13.7)$ & $0.163(14.8)$ \\
\hline$V_{z}(I)$ & $\mathrm{NC}^{n}=0$ & $21.4(15.6)$ & $14.8(13.7)$ & 19.8 (11.5) & $21.4(10.7)$ \\
\hline
\end{tabular}

Healthy subjects ( $n=6 /$ group, randomized at a $4: 2$ ratio) were dosed with $0.2,2,10,25$, or 50 mg/kg efgartigimod or placebo, respectively (i.e., cohorts 1-5). Efgartigimod or placebo was administered i.v. in a 2-hour infusion. Values are shown as arithmetic means and coefficient of variation (CV\%) in parentheses. $n$, number of subjects; $n$ (superscripted), number of subjects with data; NC, not calculated. $t_{1 / 2, \lambda z}$, apparent terminal half-life.

q7d regimen at the $10 \mathrm{mg} / \mathrm{kg}$ dose or the 10 and $25 \mathrm{mg} / \mathrm{kg}$ q7d doses in terms of $\mathrm{E}_{\max }$ or AUEC, suggesting that the maximal reduction of IgG1 levels was already reached with the $10 \mathrm{mg} / \mathrm{kg}$ dose q7d (Supplemental Table 3).

IgG2 and IgG3 subclass levels were reduced in a way over time that was similar to that of IgG1 levels, with a mean $\mathrm{E}_{\max }$ ranging from $64.6 \%$ to $76.8 \%$ for IgG2 and from $73.3 \%$ to $77.9 \%$ for IgG3. Similarly to what occurred in the SAD part, IgG4 seemed to be reduced to a slightly lesser extent, with the mean $\mathrm{E}_{\max }$ ranging from $58.1 \%$ to $66.6 \%$. The median time to maximum effect $\left(\mathrm{t}_{\mathrm{Emax}}\right)$ ranged from 14 to 27 days following the first administration dependent on the IgG subtype and on the dosing regimen. There was no significant decrease in IgA, IgD, IgE, or IgM or in albumin serum levels after administration of multiple doses of efgartigimod (Supplemental Figure 6), again demonstrating the selective IgG depletion induced by efgartigimod.

Adverse events. All efgartigimod-related treatment-emergent adverse events (TEAEs) reported in this study, grouped by system organ class and preferred term, are summarized in Table 3. Most of the efgartigimod-related adverse events (AEs) were observed in only 1 subject, mostly in the higher dose groups. Only the following AEs were reported in more than one subject: headache, chills, dizziness, fatigue, abnormal differential WBC count, and increased C-reactive protein (CRP) levels. It should be noted, however, that some of the efgartigimod-related AEs were also reported in subjects infused with placebo.

Headache was reported in 4 subjects in the SAD part of the study who received 25 or $50 \mathrm{mg} / \mathrm{kg}$ efgartigimod. In the MAD part, headache was reported in 1 subject in the $10 \mathrm{mg} / \mathrm{kg}$ q $4 \mathrm{~d}$ group and in 3 subjects in the $25 \mathrm{mg} / \mathrm{kg}$ q7d group. Apart from 1 subject in whom a moderate headache developed after single infusion of 25 $\mathrm{mg} / \mathrm{kg}$, in all other cases, this AE was considered mild. Two cases of chills were reported in the SAD part of the study in the group who received $50 \mathrm{mg} / \mathrm{kg}$ efgartigimod. The other 2 cases were reported in the MAD part in the group who received $25 \mathrm{mg} / \mathrm{kg}$ q7d. In 1 case in the $50 \mathrm{mg} / \mathrm{kg}$ group, this TEAE was considered moderate. In all other cases, it was considered mild. Dizziness related to efgartigimod, graded as mild in severity, was observed in 2 subjects in the SAD part receiving a dose of $50 \mathrm{mg} / \mathrm{kg}$. No cases of dizziness were reported in the MAD part. Fatigue was never observed in the SAD part of the study, but was reported in 2 subjects in the $25 \mathrm{mg} / \mathrm{kg}$ q7d cohort. These cases were considered mild in severity.
The most frequently reported TEAE in the SAD part was a slightly abnormal differential WBC count consisting of a mild decrease of CD8, CD3, CD56, CD4, and CD19 lymphocyte levels (7 out of 20 subjects dosed with efgartigimod). This decrease was only reported in the groups receiving a single infusion of 25 or 50 $\mathrm{mg} / \mathrm{kg}$ and was observed within the first 8 hours after delivery, with values returning to baseline 24 hours after delivery without any additional therapy. No abnormal differential WBC count was reported in the MAD part of the study. This difference is possibly due to the fact that in the MAD part, the first postdelivery measurement of lymphocyte levels was 24 hours after the first administration of the study drug, at which time the lymphocyte numbers could already have returned to baseline if an initial drop occurred.

In the SAD part of the study, a transient increase of CRP was reported (average maximum increase of $5.4 \times$ baseline value). All events of increased CRP were reported on day 2 and were considered mild or moderate in severity and related to the study drug by the investigator. All 6 subjects recovered from increased CRP within 3 to 6 days. All these subjects also presented an abnor-

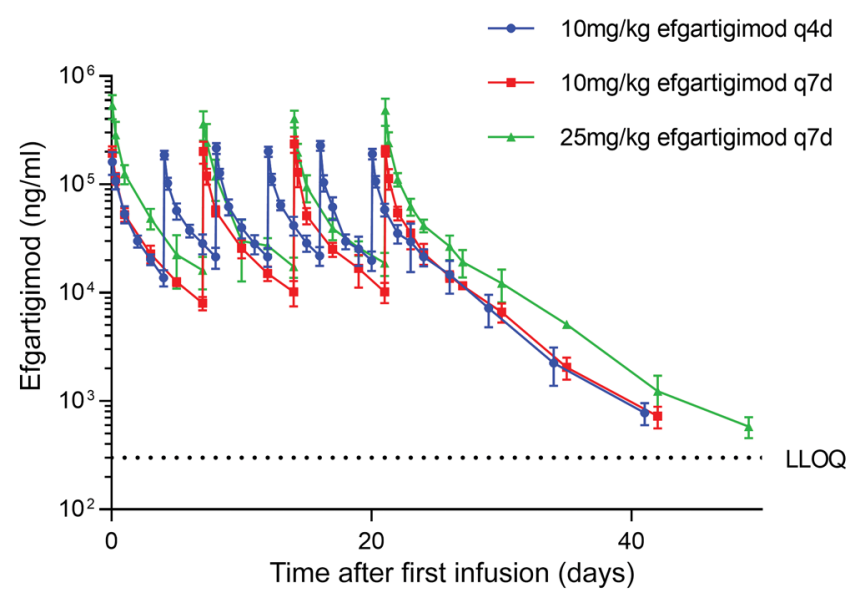

Figure 9. Efgartigimod serum concentration in the MAD part of firstin-human study. Healthy subjects ( $n=8 /$ group) were dosed with 10 $\mathrm{mg} / \mathrm{kg}$ efgartigimod or placebo q4d on 6 occasions (cohort 7), $10 \mathrm{mg} /$ $\mathrm{kg}$ efgartigimod or placebo q7d on 4 occasions (cohort 9), or $25 \mathrm{mg} / \mathrm{kg}$ efgartigimod or placebo q7d on 4 occasions (cohort 10). Efgartigimod or placebo (randomized at a 6:2 ratio) was administered i.v. in a 2-hour infusion. Values are shown as mean \pm SD. 
Table 2. Summary of efgartigimod PK parameters for MAD part

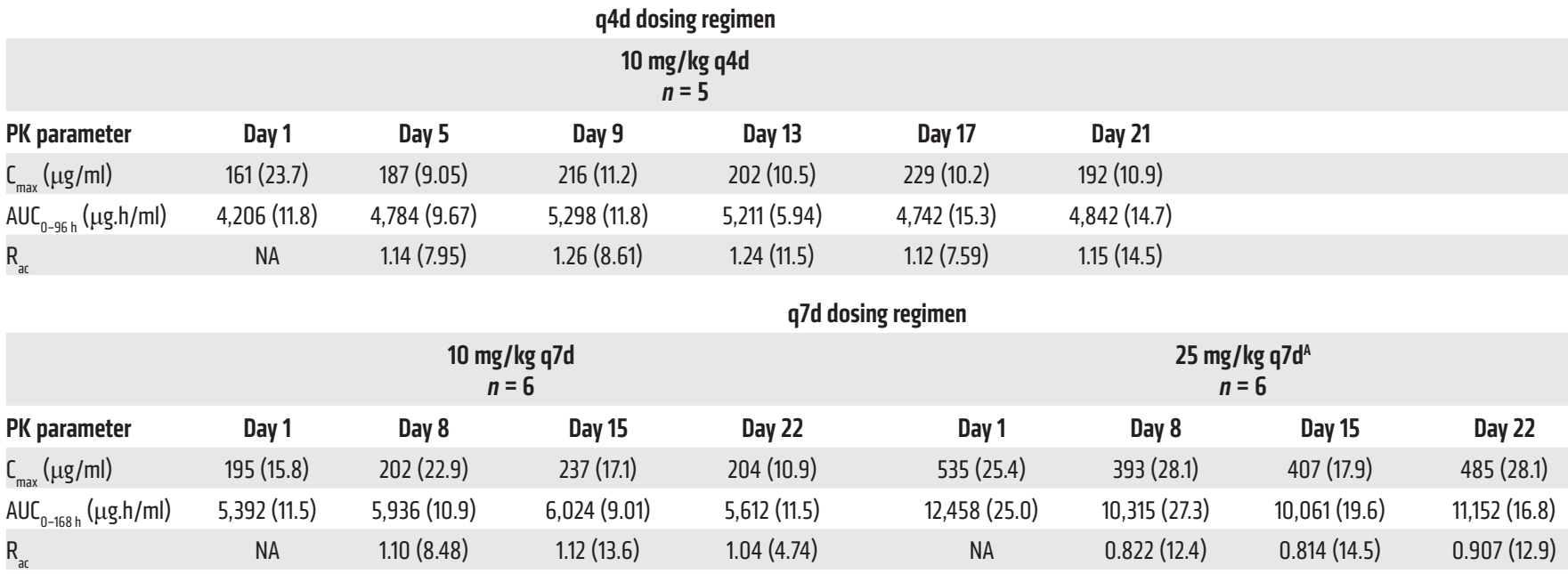

Healthy subjects ( $n=8 /$ group) were dosed with $10 \mathrm{mg} / \mathrm{kg}$ efgartigimod or placebo q4d on 6 occasions (cohort 7), $10 \mathrm{mg} / \mathrm{kg}$ efgartigimod or placebo q7d on 4 occasions (cohort 9), or $25 \mathrm{mg} / \mathrm{kg}$ efgartigimod or placebo q7d on 4 occasions (cohort 10). Efgartigimod or placebo (randomized at a 6:2 ratio) was administered i.v. in a 2-hour infusion. Values are shown as arithmetic mean (CV\% in parentheses) except geometric means (CV\% in parentheses) for $\mathrm{R}_{\text {ac }}$ ${ }^{A}$ For the $q 7 \mathrm{~d}$ regimen at the dose $25 \mathrm{mg} / \mathrm{kg}$, only the data from cohort 10 are summarized in this table. NA, not applicable.

mal differential WBC count. A mild increase (average maximum increase of $6.6 \times$ baseline value) in CRP level was also reported on day 9 in 1 subject who received 2 doses of $25 \mathrm{mg} / \mathrm{kg}$ q7d (MAD part). Except for a decrease of lymphocyte subsets and increase of CRP, no other clinically significant abnormalities of laboratory parameters were reported. Similarly, no clinically significant abnormalities of ECG were observed.

One serious adverse event (SAE) (hyperventilation) was reported in 1 subject 6 days after receiving a first infusion at a dose of 25 $\mathrm{mg} / \mathrm{kg}$. This SAE was considered unlikely to be related to the study drug. Apart from in this subject, no efgartigimod-related SAE or any other AE leading to discontinuation of treatment was reported. None of the observed AEs was certainly related to efgartigimod and, in all cases, only a possible or probable relatedness to the study drug was postulated by the investigator. Most of the AEs were observed after a single infusion of 25 or $50 \mathrm{mg} / \mathrm{kg}$ or multiple infusions of 25 $\mathrm{mg} / \mathrm{kg}$ efgartigimod. In terms of severity, most AEs reported were considered mild and, only in a few cases, moderate.

Immunogenicity in human volunteers. Overall, efgartigimod was not found to provoke the development of anti-drug Ab (ADA) signals. In a limited number of pre- and postdelivery samples originating from both active- and placebo-treated individuals, positive ADA titers were detected. These positive ADA samples were found in both the SAD and the MAD part of the study. Signals typically were just above the detection limit of the assay and were only found once during the study for most subjects. No increase of titers over time for individual subjects was observed, nor had any of the volunteers with at least 1 positive ADA sample an apparent different PK/PD profile.

\section{Discussion}

Inhibition of the IgG recycling activity of FcRn is thought to be a valid strategy to induce the clearance of pathogenic IgG autoantibodies to treat certain autoimmune diseases, representing an elegant alternative to therapeutic approaches, such as plasma exchange, IVIg treatment, or immunoadsorption. The latter approaches can result in a rapid lowering of autoantibody titers; however, they can lead to undesirable side effects and, in addition, can be cumbersome to perform. Indeed, since it became apparent that FcRn is a key mediator of IgG PK and biodistribution, various strategies have been developed to exploit its biology (24). It is established that IgG variants with altered affinity for FcRn have a longer or shorter in vivo half-life (8). The unusual $\mathrm{pH}$-dependent binding profile of FcRn-IgG interactions is a key determinant for the fate of these modified molecules. IgGs with Fc mutations that increase the affinity for $\mathrm{FcRn}$ at acidic $\mathrm{pH}$ without changing affinity at neutral $\mathrm{pH}$ typically have a longer serum half-life, while molecules having increased affinities across this $\mathrm{pH}$ range show reduced serum persistence $(12,25,26)$. By introducing Fc mutations that substantially increase the affinity at both acidic and neutral $\mathrm{pH}$, an antagonist of the recycling pathway has been generated that hijacks the natural binding site for FcRn on IgG (15). Such an antagonist outcompetes IgGs from binding to $\mathrm{FcRn}$ at acidic $\mathrm{pH}$ and, due to its increased affinity for $\mathrm{FcRn}$ at near-neutral $\mathrm{pH}$, is internalized into FcRnexpressing cells via receptor-mediated, rather than fluid-phase, uptake. However, due to the intrinsic $\mathrm{pH}$ dependence of FcRn-IgG interactions, the affinity of efgartigimod at $\mathrm{pH}$ 7.3-7.4 is approximately 20-fold lower than that at $\mathrm{pH}$ 6.0. Consistent with our earlier observations (27), this $\mathrm{pH}$ dependence enables recycling of a high proportion of the inhibitor in human FcRn-expressing cells. This concept forms the basis of the development of efgartigimod, a hIgG1-derived Fc fragment modified with ABDEG technology (15).

Consistent with previous studies using ABDEG-equipped molecules in rodents (15), efgartigimod induced an IgG-depleting effect in cynomolgus monkeys. Further, the FcRn-antagonizing potency of efgartigimod exceeded that of IVIg, as an approximately 30-fold lower dose cleared an hIgG1-based tracer Ab much more 

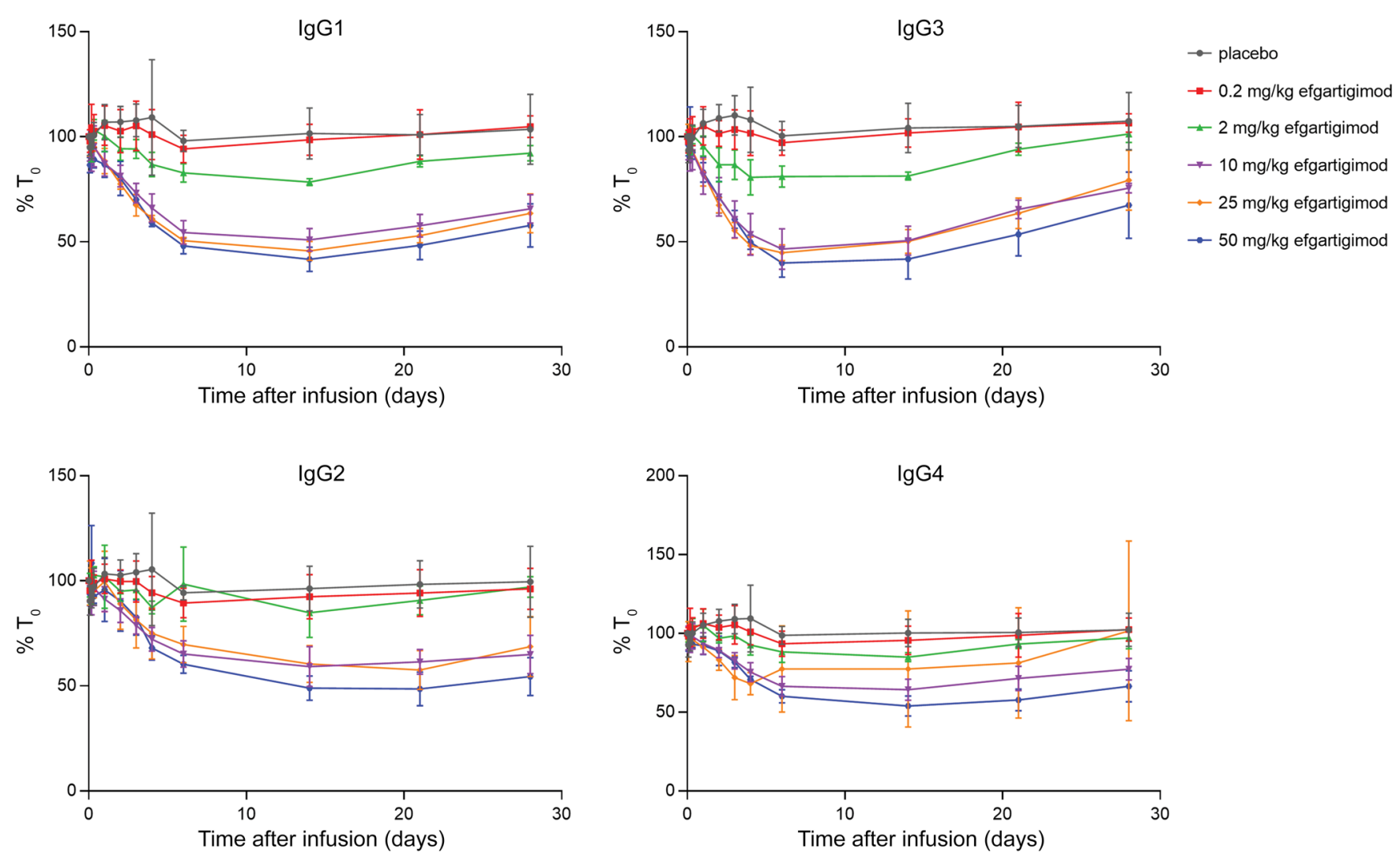

Figure 10. Serum levels of IgG subclasses over time in the SAD part of first-in-human study. Healthy subjects $(n=6 /$ group) were dosed with $0.2,2,10$, 25 , or $50 \mathrm{mg} / \mathrm{kg}$ efgartigimod or placebo (i.e., cohort 1-5). Efgartigimod or placebo (randomized at a 4:2 ratio) was administered i.v. in a 2-hour infusion. Percentage change versus baseline in $\operatorname{IgG}_{\text {subclass }}$ serum concentration $\left(\% \mathrm{~T}_{0}\right)$ is shown. Values are shown as mean $\pm \mathrm{SD}$.

effectively. The specific and rapid IgG-depleting effect of this firstin-class FcRn antagonist was confirmed in a first-in-human study in 62 healthy volunteers. Serum IgG levels decreased on average by approximately $75 \%$ upon multiple doses of efgartigimod, with some individuals showing reductions of up to $85 \%$. This nadir is similar to the low IgG titers observed in 2 patients with familial hypercatabolic hypoproteinemia due to aberrant FcRn expression $(28,29)$, indicating that further infusion of an $\mathrm{FcRn}$ antagonist would not result in more profound lowering of IgG levels. Interestingly, for all volunteers administered with efgartigimod at doses equal to or above $10 \mathrm{mg} / \mathrm{kg}$, a clear IgG reduction was detected, and although a large intersubject difference in absolute baseline IgG levels was observed, relative reductions were remarkably consistent between subjects. When dosing was stopped, IgG levels started to recover and gradually returned to baseline levels toward the end of the study ( $>50$ days following the last infusion). This sustained PD effect contrasts with the PK profile of efgartigimod, showing a rapid reduction in serum concentrations of the compound following the last dosing. These findings suggest that most of the administered efgartigimod is rapidly bound to FcRn, antagonizing its function for a prolonged period, and this hypothesis is supported by in vitro analysis showing efficient drug accumulation in FcRn-expressing cells. The FcRn-based recycling observed for efgartigimod could further contribute to a sustained PD effect, as 1 efgartigimod molecule in theory would be capable of antagonizing FcRn function during multiple recycling rounds.
This favorable PK/PD profile could support treatment of various autoimmune diseases using efgartigimod. The current PD data additionally do not suggest an overshoot of IgG titers above baseline levels following efgartigimod treatment, as reported for plasma exchange (30), although the study duration could have been too short to fully exclude this phenomenon. Interestingly, the reduction of the levels of the different IgG subtypes $(1,2,3$, and 4$)$ over time was very similar, although IgG4 seemed to be reduced to a slightly lesser extent. This could suggest a different production rate or a different $\mathrm{FcRn}$ interaction profile of IgG4 compared with the other IgG subtypes, although the latter hypothesis is not supported experimentally $(31,32)$.

Efgartigimod administration did not result in significant changes in IgA, IgD, IgE, and IgM levels. This was expected, since the endogenous levels of these Igs are not dependent on FcRnmediated recycling. Therefore, targeted depletion of IgGs by efgartigimod could signify a clear safety advantage over techniques such as plasma exchange, which removes all serum proteins and hence results in broader immunosuppression and increased infection risk. Efgartigimod also did not influence albumin homeostasis in the animal and the phase I study. This finding indicates that efgartigimod administration does not increase the turnover rate of FcRn. Our in vitro analyses indicate that an anti-FcRn Ab, with higher affinity binding than efgartigimod to FcRn at both nearneutral $\mathrm{pH}$ and acidic $\mathrm{pH}$, shows higher levels of accumulation/ retention and lysosomal localization in receptor-expressing cells. 

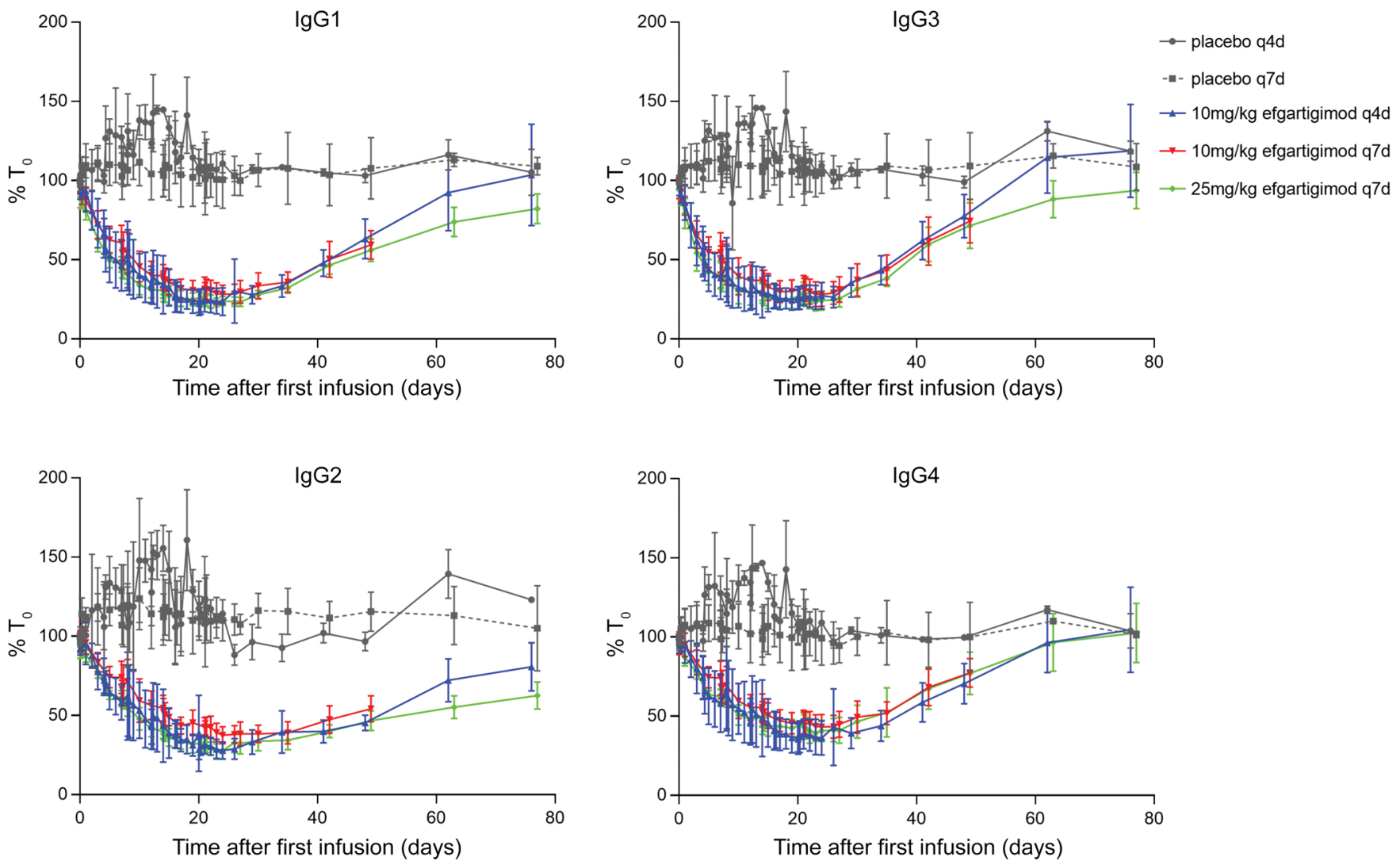

Figure 11. Serum levels of IgG subclasses over time in the MAD part of first-in-human study. Healthy subjects ( $n=8 /$ group) were dosed with $10 \mathrm{mg} /$ $\mathrm{kg}$ efgartigimod or placebo q4d on 6 occasions (cohort 7), $10 \mathrm{mg} / \mathrm{kg}$ efgartigimod or placebo q7d on 4 occasions (cohort 9), or $25 \mathrm{mg} / \mathrm{kg}$ efgartigimod or placebo q7d on 4 occasions (cohort 10). Efgartigimod or placebo (randomized at a 6:2 ratio) was administered i.v. in a 2-hour infusion. Percentage change versus baseline in Ig $\mathrm{G}_{\text {subclass }}$ serum concentration $\left(\% \mathrm{~T}_{0}\right)$ is shown. Values are shown as mean $\pm \mathrm{SD}$.

In contrast with efgartigimod, this $\mathrm{Ab}$ has $4 \mathrm{FcRn}$ interaction sites per molecule ( $2 \mathrm{Fab}, 2 \mathrm{Fc}$ ). Earlier studies have demonstrated that crosslinking of FcRn by multivalent immune complexes can result in both increased lysosomal trafficking of FcRn-ligand complexes and immune activation $(33,34)$, and further studies are necessary to determine how affinity and/or valency of the FcRn-inhibitor complex affects intracellular trafficking and cellular responses.

The extent of the efgartigimod-induced IgG lowering is similar or exceeds the levels of autoantibody reduction achieved with alternative strategies, such as IVIg, plasma exchange, or immunoadsorption, and falls within the range associated with a clear clinical benefit in various autoimmune indications (35-37). Indeed, reducing the autoantibody levels in myasthenia gravis patients by about 30\% using IVIg resulted in a clinical efficacy rate of $40 \%$ (35), and this efficacy rate increased to $80 \%$ when titers were further lowered by plasma exchange to $60 \%$. Interestingly, the human PD effects seemed to be more pronounced than in nonhuman primates, as the IgG depletion was more sustained with a 2-fold lower saturating dose $(10 \mathrm{mg} / \mathrm{kg}$ versus $20 \mathrm{mg} / \mathrm{kg}$ in cynomolgus monkeys). Administration i.v. of efgartigimod in healthy subjects was associated with a few, mostly mild and self-limiting drug-related AEs, the majority of which were reported only at the highest doses.

Except for 2 incidents of rash in the MAD part of the study, infusion-related or injection site AEs typically observed with i.v. biologic therapies were not observed after efgartigimod dos- ing. This could potentially be explained by the reduced affinity of efgartigimod for Fc $\gamma$ Rs due to the introduction of the ABDEG mutations (38) and the favorable physicochemical characteristics of the molecule with a low percentage of aggregates in the drug product. Collectively, these factors are expected to reduce the risk of unwanted immune stimulation.

It is possible that a reduction of IgG levels would increase the risk of developing infections (39). In the current study in human volunteers, however, a higher rate of infections was not observed following exposure at any level to efgartigimod. The observation that efgartigimod results in a maximum decrease of the IgG level of around $75 \%$ to $85 \%$ and does not affect other Ig isotypes may explain the absence of an increased infection rate.

Clinically mild headache was observed in 4 subjects and moderate headache in 1 subject following treatment with efgartigimod. As headache is commonly observed in patients after infusion of therapeutic Abs $(40,41)$, it is not unexpected that headache was reported in a few subjects. However, it should be noted that efgartigimod-related headache was only reported in the groups exposed to the highest doses. Contrary to observations with other therapeutic Abs and IVIg where this AE is very common and severe, frequently requiring treatment with potent antimigraine and pain-killing drugs (42), the headache reported after infusion of efgartigimod was mostly mild and resolved quickly and spontaneously or after treatment with over-the-counter medications. 


\section{Table 3. Number of subjects with AEs observed in phase I healthy volunteers study}

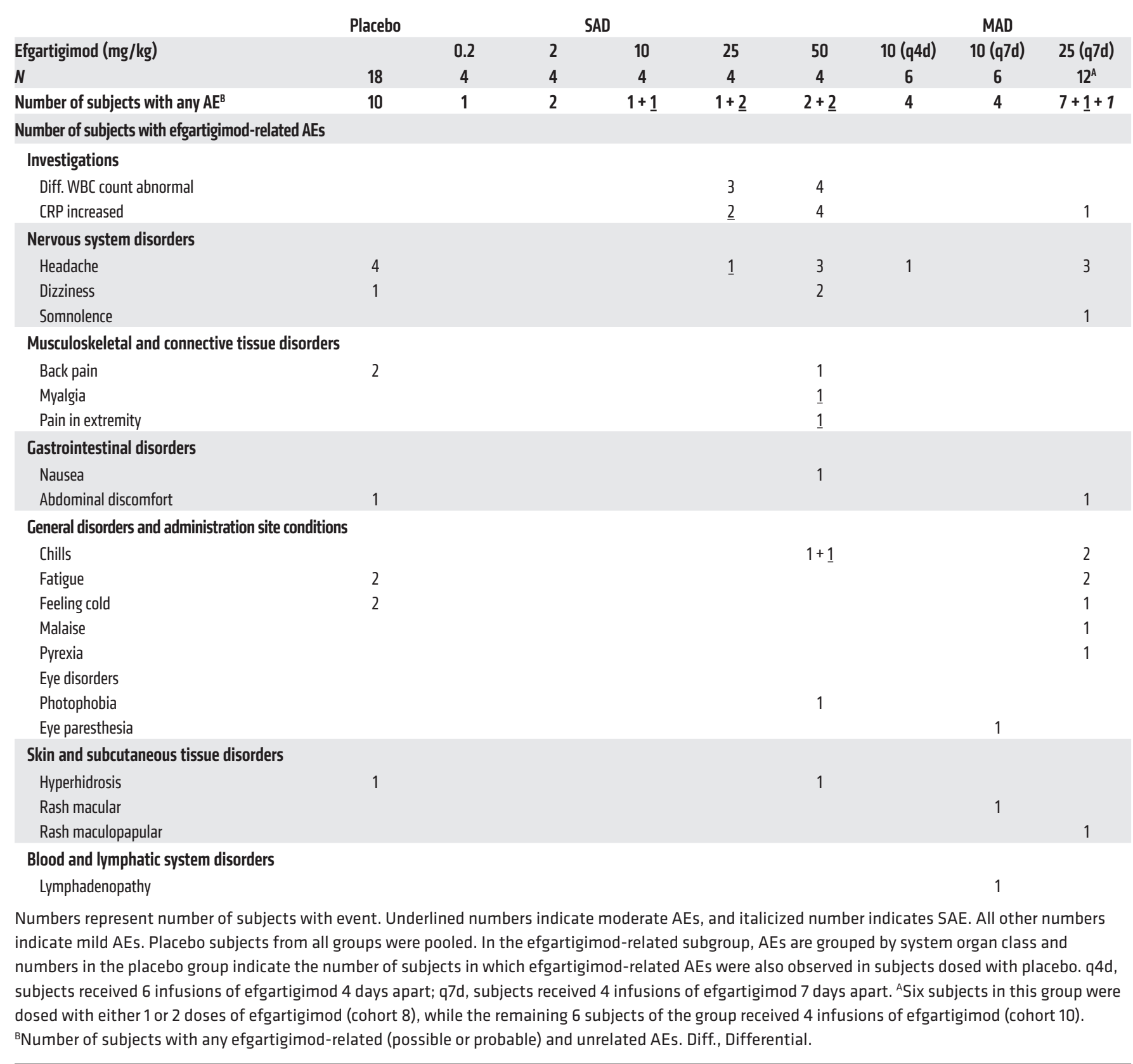

Taken together, these data are consistent with previous reports attributing the rapidly occurring headaches following IVIg treatment to the peak IgG concentration $(43,44)$ and suggest that the mild headaches observed with high-dose efgartigimod could also be related to peak concentration rather than to an FcRn-related phenomenon. Indeed, FcRn blockade is nearly maximal at a dose of $10 \mathrm{mg} / \mathrm{kg}$, while headaches were primarily observed in the higher dose cohorts. Moreover, IVIg infusion can result in several side effects, such as infusion site reactions, neurological and renal complications, and thrombotic events. Some of these side effects can be serious and even life threatening (44). In this regard, given its benign safety profile in human volunteers, efgartigimod may represent a much safer alternative to IVIg for treating IgG-mediated autoimmune diseases. In addition, the maximum decrease of IgG levels was observed after multiple infusions of $10 \mathrm{mg} / \mathrm{kg}$ efgartigimod. Higher doses did not result in significant further reductions of IgG levels, while at the same time, the number of subjects experiencing efgartigimod-related AEs increased. Therefore, the dose of $10 \mathrm{mg} / \mathrm{kg}$ administered weekly seems to provide the best benefit to risk ratio and was selected for future studies in patients.

The current phase I clinical study proved that inhibition of FcRn in humans leads to a rapid, specific, deep, and prolonged reduction of IgG serum levels. The promising properties and favorable safety profile of efgartigimod reported here provide the basis for studying this agent in patients suffering from IgG-driven autoimmune indications, such as pemphigus vulgaris, primary 
immune thrombocytopenia, or myasthenia gravis. In these patient studies, it will be assessed whether administration of a repeated dose of $10 \mathrm{mg} / \mathrm{kg}$ efgartigimod results in a similar reduction of autoantibodies compared with the observed PD effects in healthy volunteers and, if so, whether that results in an alleviation of the signs and symptoms of these autoimmune patients.

\section{Methods}

Abs. Efgartigimod used in nonclinical experiments was transiently produced in CHO cells and purified using MabSelect SuRe resin (Evitria). Efgartigimod for human use was produced by Lonza Biologics using a CHOK1SV GS-KO cell line (Lonza Group Ltd.) stably transfected with an expression vector encoding efgartigimod. Purification was performed in a 3-step process including protein A column chromatography, anion exchange chromatography, and hydrophobic interaction chromatography. Production yield and glycosylation profile were in line with what is typically observed for full-length IgG Abs expressed using this system.

FR70 $\mathrm{Ab}$ is a rat anti-mouse CD70 mAb produced by hybridoma technology (45). Following sequencing of its variable regions using standard procedures, mammalian expression vectors were made encoding FR70-hIgG1. Subsequently, FR70-hIgG1 was produced transiently in HEK293-E cells and purified using protein A beads at U-Protein Express. IVIg (Octagam 10\%) was bought from Octapharma. An anti-FcRn Ab (DX-2507) was previously described (18), and sequences for the heavy and light chains were retrieved from patent application WO2012/167039. cDNA sequences for the heavy and light chains of the Ab were cloned into mammalian expression vectors. Subsequently, the anti-FcRn Ab was produced and purified as described for FR70-hIgG1. The humanized anti-HEL Ab (hIgG1) was expressed and purified from culture supernatants of transfected NSO cells using HEL-sepharose and previously described methods (46).

For in vitro use, Abs or efgartigimod was labeled with Alexa Fluor $647 \mathrm{~N}$-hydroxysuccinimide (NHS) ester, tris(triethylammonium salt) (Invitrogen, Thermo Fisher Scientific), using methods recommended by the manufacturer, except that the molar ratio of dye:Ab/Fc fragment was 2:1. Degrees of labeling for each $\mathrm{Ab} / \mathrm{Fc}$ fragment were as follows: efgartigimod, 2.2 dye:Fc; hIgG1, 1.7 dye:Ab; anti-FcRn, and 1.45 dye:Ab. Labeled $\mathrm{Ab} / \mathrm{Fc}$ fragments were analyzed to ensure that there were no detectable aggregates using HPLC (Superdex 200 or Yarra 3 $\mu \mathrm{m}$ SEC 3000).

Anti-CD4-Pacific Blue (BD Biosciences - Pharmingen, clone RPAT4), anti-CD45-Krome Orange (Beckman Coulter, clone J.33), antiCD8 FITC (Dako, clone DK25), anti-CD56-PE (BD, clone NCAM16.2), anti-CD16-PE (BD, clone Leu 11c), anti-CD19-PC7 (Beckman Coulter, clone J3.119), and anti-CD3-APC (BD, clone SK7) were used for flow cytometric analysis of the human lymphocyte subsets. The dermalderived microvasculature cell line HMEC-1.CDC was provided by F. Candal (CDC, Atlanta, Georgia, USA).

FcRn expression. Recombinant human FcRn for surface plasmon resonance analyses was expressed and purified using previously described methods (47). Biotinylated recombinant cynomolgus monkey and human FcRn used in FcRn-binding ELISA were bought from Immunitrack (catalog ITF05 and ITF01, respectively).

Surface plasmon resonance analyses. Analyses of the binding of human FcRn to efgartigimod or anti-FcRn Ab were carried out using BIAcore T200 according to previously described methods (48). Efgar- tigimod or anti-FcRn Ab was immobilized on CM5 sensor chips using amine coupling chemistry, and a flow cell treated with buffer during coupling was used as reference. FcRn was injected over the flow cells at different concentrations in PBS, $\mathrm{pH} 6.0$ or $\mathrm{pH} 7.4$ plus $0.05 \%$ Tween 20 at a flow rate of 5 or $10 \mu \mathrm{l} /$ minute. Flow cells were regenerated at the end of each cycle by injecting $0.1 \mathrm{M} \mathrm{NaHCO}_{3}, 0.1 \mathrm{M} \mathrm{NaCl}, \mathrm{pH} 8.5$ (efgartigimod), or 10 mM glycine, pH 1.8 (anti-FcRn). Equilibrium dissociation constants were determined using a custom written software SPRTool and previously described methods (http://www.wardoberlab. com/software/sprtool/) $(49,50)$.

FcRn-binding ELISA. Biotinylated human or cynomolgus monkey FcRn was captured on Pierce neutravidin-coated MaxiSorp plates (Thermo Fisher Scientific, catalog 31000), after which binding of efgartigimod and the WT Fc fragment were measured in ELISA at both pH 6.0 and pH 7.4 using HRP-labeled (Fab')2 directed against anti-hFc (Abcam, catalog ab98595).

Flow cytometry analyses. HMEC-1 cells were maintained in phenol red-free Ham's F-12K medium prior to transfection. Cells were transiently transfected with expression plasmids encoding human FcRn-GFP and $\beta 2$ microglobulin using Nucleofector technology as described previously $(10,11)$ and plated in 24 -well plates. Eighteen hours after transfection, cells were pulsed with $25 \mu \mathrm{g} / \mathrm{ml}$ Alexa Fluor 647-labeled efgartigimod, $75 \mu \mathrm{g} / \mathrm{ml}$ Alexa Fluor 647-labeled antiFcRn Ab, or $200 \mu \mathrm{g} / \mathrm{ml}$ Alexa Fluro 647-labeled hIgG1 (anti-HEL) in medium adjusted to $\mathrm{pH} 7.4$ for 30 minutes at $37^{\circ} \mathrm{C}$ in a $5 \% \mathrm{CO}_{2}$ incubator, washed, and immediately fixed using $1.7 \%$ paraformaldehyde $/ 0.025 \%$ glutaraldehyde on ice for 10 minutes or transferred to medium ( $\mathrm{pH} 7.4$ ) and incubated at $37^{\circ} \mathrm{C}$ in a $5 \% \mathrm{CO}_{2}$ incubator for 30 or 120 minutes. Following the different chase periods, cells were washed and fixed. Levels of Alexa Fluor 647 remaining in the cells were analyzed using an Accuri C6 Flow Cytometer (BD Biosciences) and data processed, including determination of mean fluorescence intensities (MFIs) using FlowJo software.

Fluorescence microscopy. HMEC-1 cells were treated as for flow cytometry, except that they were plated in MatTek dishes fitted with cover glasses (Electron Microscopy Sciences, no. 1.5, $22 \mathrm{~mm}$ diameter) following transfection. Transfected cells were pulsed with $500 \mu \mathrm{g} / \mathrm{ml}$ Alexa Fluor 555-labeled $10 \mathrm{kDa}$ dextran (Invitrogen) for 1 hour at $37^{\circ} \mathrm{C}$ in a $5 \% \mathrm{CO}_{2}$ incubator, then washed and chased for 4 hours to label lysosomes. Cells were then pulsed with labeled Abs/efgartigimod as for flow cytometry, chased in medium (pH 7.4) for 0 (i.e., no chase) or 16 hours at $37^{\circ} \mathrm{C}$ in a $5 \% \mathrm{CO}_{2}$ incubator, washed, and fixed as described above, except that fixation was carried out at room temperature.

Following fixation, cells were imaged using a Zeiss (Axio Observer Z1) inverted epifluorescent microscope equipped with a $\times 63,1.4$ NA Plan-Apochromat objective (Carl Zeiss), a ×1.6 internal optovar, and a cooled CCD camera (Orca ER, Hamamatsu). A broadband LED lamp (X-Cite 110LED, Excelitas Technologies) was used as the excitation source, and standard fluorescent filter sets for GFP (FITC-3540C-ZHE M327120), Alexa Fluor 555 (Cy3-4040C-ZHE M327122), and Alexa Fluor 647 (Cy5-4040C-ZHE M327126) were used to acquire the images. All filter sets were purchased from Semrock. Image acquisition, control, and time synchronization of the optical components of the microscope setup were performed through custom software written in Java and C programming languages. All data were processed and displayed using in-house written software (MIATool) (51). For display purposes, independent linear adjust- 
ments were made for the Alexa Fluor 555 (dextran) channel. The images acquired for Alexa Fluor 647-labeled IgG or Fc or FcRn-GFP shown in Figure 2 are displayed as acquired or as indicated in the legend for Supplemental Figure 3. Fluorescence intensity plots show the pixel intensities along the indicated lines in the figures.

Clearance of human FR7O-hIgG1 tracer Ab by efgartigimod and IVIg in cynomolgus monkeys. Captive-bred cynomolgus monkeys were ordered from R.C. Hartelust BV. Upon arrival at the test facility (LPT, Hamburg, Germany), the animals were subjected to a veterinary examination and allowed to acclimatize for a period of 4 weeks before initiation of the study. Body weight of the animals ranged from 2.5 to $3.4 \mathrm{~kg}$.

Cynomolgus monkeys ( $n=2$ /group) were administered with 1 $\mathrm{mg} / \mathrm{kg}$ tracer $\mathrm{Ab}$ via i.v. bolus injection 2 days prior to i.v. infusion of $70 \mathrm{mg} / \mathrm{kg}$ efgartigimod or $2 \mathrm{~g} / \mathrm{kg}$ IVIg.

Blood was collected from the saphena magna of all animals and centrifuged to obtain serum. Tracer Ab levels in blood serum were determined by murine CD70-binding ELISA using MaxiSorp plates (Thermo Fisher Scientific, catalog 31000) and murine CD70 (R\&D Systems, cata$\log 783-\mathrm{CL} / \mathrm{CF}$ ). Tracer levels were plotted relative to predose levels.

$S A D$ and MAD studies in cynomolgus monkeys. Cynomolgus monkeys ( $n=2 /$ group) were given an i.v. bolus injection of $1 \mathrm{mg} / \mathrm{kg}$ of tracer FR70-hIgG1. After 48 hours, animals were infused with a single dose of efgartigimod (at 0.2, 2, 20, or $200 \mathrm{mg} / \mathrm{kg}$ ) or vehicle (PBS).

Levels of tracer $\mathrm{Ab}$ in blood serum were determined by ELISA as described above. Endogenous IgG was detected by ELISA using an anti-monkey IgG- $\gamma$-chain-specific capturing Ab (Sigma Aldrich, catalog SAB3700764) and an HRP-coupled mouse anti-monkey IgG detection $\mathrm{Ab}$ (Southern Biotech, catalog 4700-05). Tracer levels were plotted relative to predose levels. For the $200 \mathrm{mg} / \mathrm{kg}$ efgartigimod group, IgA and IgM levels were additionally determined using a commercial ELISA kit (Alpha Diagnostics, catalog 7010 and 7060, respectively). No tracer was detected in any of the serum samples of 1 animal dosed with $2 \mathrm{mg} /$ $\mathrm{kg}$ efgartigimod, and therefore data from this animal were not included.

In the multiple dose study, 1 cohort of 2 cynomolgus monkeys received an efgartigimod infusion at $20 \mathrm{mg} / \mathrm{kg}$ every 24 hours during the first 4 days, while the other cohort of 2 monkeys received the same dose once every 4 days (q4d). Determination of endogenous IgG levels was performed as described above.

Human volunteer trial: phase I study design. From September 30, 2015, to October 14, 2016, we performed a first-in-human, randomized, double-blind, placebo-controlled, single-center study to evaluate i.v. SAD and MAD of efgartigimod in 62 healthy subjects. The study was done by SGS Belgium (Antwerp, Belgium) and consisted of 2 parts: (a) a SAD part in 30 subjects and (b) a MAD part in 32 subjects.

The primary objective of the study was to evaluate the safety and tolerability of efgartigimod as compared with placebo. The secondary objectives were the determination of PK parameters, PD effects, and immunogenicity of the drug.

The SAD part was conducted in 5 sequential (dose level) cohorts (cohorts 1-5), each of which consisted of 6 subjects randomized to receive efgartigimod or placebo at a 4:2 ratio. The MAD part consisted of 4 cohorts (cohorts 7-10) of 8 healthy subjects each, who were randomized to receive multiple doses of efgartigimod or placebo at a 6:2 ratio.

Allocation of each subject to a given treatment was described in a randomization list prepared by SGS Life Sciences Secure Data Office (SGS LS SDO) using SAS software (SAS Inc.). An unblinded pharmacist or appropriate qualified member of the study staff prepared the study drug that corresponded to the assigned subject randomization number. The subjects, clinical study staff, and sponsor were blinded to treatment. The placebo contained the same formulation as efgartigimod, but without active ingredient, and matched efgartigimod in appearance. No formal sample size calculations were performed.

In the SAD part (cohorts 1-5), subjects were dosed with 0.2, 2, 10, 25 , or $50 \mathrm{mg} / \mathrm{kg}$, respectively. Efgartigimod or placebo was administered i.v. in a 2-hour infusion. Demographic data and baseline characteristics are displayed in Supplemental Table 4. Dose escalation was dependent upon satisfactory review of the blinded safety data by the safety committee, consisting of, but not limited to, the sponsor's medical monitor and the principal investigator of the study (SGS Belgium). Subjects enrolled in the MAD part were dosed by means of an i.v. infusion over 2 hours as follows: $10 \mathrm{mg} / \mathrm{kg}$ efgartigimod or placebo $\mathrm{q} 4 \mathrm{~d}$ on 6 occasions (cohort 7), $25 \mathrm{mg} / \mathrm{kg}$ efgartigimod or placebo q7d on 4 occasions (cohort 8), $10 \mathrm{mg} / \mathrm{kg}$ efgartigimod or placebo q7d on 4 occasions (cohort 9), or $25 \mathrm{mg} / \mathrm{kg}$ efgartigimod or placebo q7d on 4 occasions (cohort 10). Dose-escalation procedures were similar to those in the SAD part. Dosing of all subjects in cohort 8 was discontinued early because of 1 subject experiencing an SAE. At that time, 4 subjects in this cohort had received 2 doses of the study drug, and 4 subjects, including the subject with the SAE, had received 1 dose of the study drug. These subjects were not included in the PK and PD evaluation. Demographic data and baseline characteristics of the MAD cohorts are displayed in Supplemental Tables 5 and 6. Eligible subjects were males or females of nonchildbearing potential, aged between 18 and 55 years, with a body mass index between 18 and $30 \mathrm{~kg} / \mathrm{m}^{2}$ and a weight between 50 and 100 $\mathrm{kg}$ and were in good health. Major exclusion criteria included the usual criteria used in phase I settings, such as major ongoing infections, hypersensitivities to any ingredient subjects were to be exposed to, and a history of malignancies. Protocol synopsis, including schedule of assessments, can be found in the Supplemental Protocol Synopsis. Off-note, at the time of study initiation, the international nonproprietary name (INN) efgartigimod was not yet available, and hence the compound was called by its product code, ARGX-113, throughout the protocol.

$P K, P D$, immunogenicity, and lymphocyte analysis. PK, PD, and ADA assay validation and sample analysis were performed by SGS Céphac (Saint-Benoît, France). Serum levels of efgartigimod were determined by means of a validated ELISA method using a proprietary efgartigimod-specific capturing Ab (FairJourney Biologics). The lower limit of quantification of the assay was $300 \mathrm{ng} / \mathrm{ml}$. In the SAD part of the study, urine levels of efgartigimod were analyzed up to 72 hours after infusion. PK calculations were performed using Phoenix WinNonLin, version 6.2 or higher (Pharsight Corp.), which was applied using a noncompartmental method. $\mathrm{R}_{\text {ac }}$ after dosing cycle $\mathrm{x}$ was defined as $\mathrm{AUC}_{\text {dosing interval } \mathrm{x}} / \mathrm{AUC}_{\text {dosing interval } 1}$.

IgG subclasses (1, 2, 3, and 4) and IgA and IgM levels were determined via validated analytical methods using a Luminex Multiplex Immunoassay (Bio-Plex Pro Human Isotyping Panel Kit; Bio-Rad Laboratories, catalog 171-A3100M), while IgD levels were measured via an ELISA using commercially available reagents (Human IgD ELISA Quantitation Set, Bethyl Laboratories Inc., catalog E80-106-13). The analysis of study samples for IgE was performed using clinically validated, commercially available packs IgE II (catalog 04827031190), IgE CalSet (catalog 11930427122), and PreciControl Universal (cata$\log 11731416190)$ (Roche Diagnostics). PD parameters were calculated using Phoenix WinNonLin, version 6.2 or higher (Pharsight Corp.). 
Determination of ADA levels was performed with a validated sandwich ELISA method using unconjugated efgartigimod as capturing agent and biotinylated efgartigimod for detection. Samples scoring positive in a screening ELISA were subjected to a confirmatory assay, which assessed the specificity of the ADA response for efgartigimod. When specificity was demonstrated, a titration of the ADA response was performed. Neutralizing potency of ADA response was not assessed. Leukocyte immunophenotyping was performed by flow cytometry following version 7 of the lymphoid screening tube (LST) strategy (52) and using Abs outlined above.

Safety. The safety assessments were based on AE reporting. All AEs starting or worsening on or after first dosing were considered TEAEs. For each AE, severity (mild, moderate, or severe) and causality (unrelated, unlikely, possibly, probably, or certainly related) were assessed by the investigator. TEAEs were considered related to the study drug when classified as possibly, probably, or certainly related.

Statistics. Descriptive statistics were used in the nonclinical animal studies. Flow cytometry data were analyzed by 1-way ANOVA $(P<0.05$ was considered significant). For the PK analysis of the healthy volunteer data, descriptive statistics were calculated by dose (SAD part) and by dose and day (MAD part) for the serum concentrations and PK parameters of efgartigimod. Dose proportionality was assessed in the SAD part only across efgartigimod doses using the power model described by Gough et al. (53), which was applied to the log-transformed $\mathrm{AUC}_{\text {inf }}$ and $\mathrm{C}_{\text {max }}$ data. A point estimate and 90\% CI were determined for the population mean slope. Approximate dose proportionality was concluded if the $90 \%$ CI for the slope was completely contained in the range $(1+\log (0.5) / \log (r), 1+\log (2) /$ $\log (r))$, where $r=$ high dose/low dose. For the PD data, the AUECs and $\mathrm{E}_{\max }$ were compared between the treatment groups (placebo subjects pooled) using a 1-way ANOVA model with treatment as a fixed effect $(P<0.05$ was considered significant). In the case of a significant treatment effect, pairwise comparison between treatments was performed using Tukey's test. As $\mathrm{t}_{\mathrm{Emax}}$ is a discrete variable dependent on selected blood sampling times, comparison between treatment groups (placebo subjects pooled) was assessed by using a nonparametric Kruskal-Wallis test and, in cases in which it was statistically significant $(P<0.05)$, by applying Wilcoxon's rank sum test with Moses' $90 \%$ CIs for pairwise comparisons. Safety parameters were tabulated and analyzed descriptively.

Study approval. Cynomolgus monkey study protocols were conducted according to German regulations and were approved by the local government (Niedersächsisches Landesamt für Verbraucherschutz und Lebensmittelsicherheit, Wardenburg, Germany). For the first-in-human study, the Belgian Regulatory Agency and the local Ethics Committees approved the protocol, and the study was conducted in accordance with the Declaration of Helsinki and in compliance with the ICH E6 GCP guidelines. The study was registered on www. clinicaltrials.gov (NCT03457649). All subjects gave written informed consent prior to inclusion in the study.

\section{Author contributions}

PU, AG, TD, HDH, ESW, and NL wrote and revised the manuscript. PU, TD, EH, ESW, RJO, PV, and HDH designed and supervised the nonclinical studies, and $\mathrm{BV}, \mathrm{VH}, \mathrm{NO}, \mathrm{VL}$, and JC acquired the nonclinical data. PU, AG, FJE, TVB, TD, and NL wrote the clinical study protocol and supervised the study. PU, AG, TD, HDH, ESW, $\mathrm{EH}$, and NL analyzed and interpreted data.

\section{Acknowledgments}

We thank Sreevidhya Ramakrishnan and Sungyong You for assistance with the microscopy experiments, Anna Hultberg for carefully reading the manuscript, and Luc Geeraert (Bench to Pen) for assistance in the writing of the manuscript.

Address correspondence to: Nicolas Leupin, argenx BVBA, Industriepark 7, 9052 Zwijnaarde, Belgium. Phone: 32.9.310.34.35; Email: Nleupin@argenx.com.
1. Cooper GS, Bynum ML, Somers EC. Recent insights in the epidemiology of autoimmune diseases: improved prevalence estimates and understanding of clustering of diseases. JAutoimmun. 2009;33(3-4):197-207.

2. Sinha AA, Lopez MT, McDevitt HO. Autoimmune diseases: the failure of self tolerance. Science. 1990;248(4961):1380-1388.

3. Sesarman A, Vidarsson G, Sitaru C. The neonatal Fc receptor as therapeutic target in IgGmediated autoimmune diseases. Cell Mol Life Sci. 2010;67(15):2533-2550.

4. Bambauer R, Latza R, Burgard D, Schiel R. Therapeutic apheresis in hematologic, autoimmune and dermatologic diseases with immunologic origin. Ther Apher Dial. 2016;20(5):433-452.

5. Perez EE, et al. Update on the use of immunoglobulin in human disease: A review of evidence. JAllergy Clin Immunol. 2017;139(3S):S1-S46.

6. Low SC, Mezo AR. Inhibitors of the FcRn:IgG protein-protein interaction. AAPS J.

2009;11(3):432-434.

7. Brambell FW. The transmission of immunity from mother to young and the catabolism of immunoglobulins. Lancet. 1966;2(7473):1087-1093.

8. Challa DK, Velmurugan R, Ober RJ, Sally Ward E.
FcRn: from molecular interactions to regulation of IgG pharmacokinetics and functions. Curr Top Microbiol Immunol. 2014;382:249-272.

9. Roopenian DC, Akilesh S. FcRn: the neonatal Fc receptor comes of age. Nat Rev Immunol. 2007;7(9):715-725.

10. Ober RJ, Martinez C, Lai X, Zhou J, Ward ES. Exocytosis of IgG as mediated by the receptor, FcRn: an analysis at the single-molecule level. Proc Natl Acad Sci U S A. 2004;101(30):11076-11081.

11. Ober RJ, Martinez C, Vaccaro C, Zhou J, Ward ES. Visualizing the site and dynamics of IgG salvage by the MHC class I-related receptor, FcRn. J Immunol. 2004;172(4):2021-2029.

12. Ghetie V, Hubbard JG, Kim JK, Tsen MF, Lee Y, Ward ES. Abnormally short serum half-lives of IgG in beta 2-microglobulin-deficient mice. Eur J Immunol. 1996;26(3):690-696.

13. Roopenian DC, et al. The MHC class I-like IgG receptor controls perinatal IgG transport, IgG homeostasis, and fate of IgG-Fc-coupled drugs. J Immunol. 2003;170(7):3528-3533.

14. Sand KM, Bern M, Nilsen J, Noordzij HT, Sandlie I, Andersen JT. Unraveling the interaction between FcRn and albumin: opportunities for design of albumin-based therapeutics. Front
Immunol. 2014;5:682.

15. Vaccaro C, Zhou J, Ober RJ, Ward ES. Engineering the Fc region of immunoglobulin $\mathrm{G}$ to modulate in vivo antibody levels. Nat Biotechnol. 2005;23(10):1283-1288.

16. Challa DK, et al. Autoantibody depletion ameliorates disease in murine experimental autoimmune encephalomyelitis. MAbs. 2013;5(5):655-659.

17. Patel DA, Puig-Canto A, Challa DK, Perez Montoyo H, Ober RJ, Ward ES. Neonatal Fc receptor blockade by Fc engineering ameliorates arthritis in a murine model.JImmunol. 2011;187(2):1015-1022.

18. Nixon AE, et al. Fully human monoclonal antibody inhibitors of the neonatal fc receptor reduce circulating IgG in non-human primates. Front Immunol. 2015;6:176.

19. Gan Z, Ram S, Ober RJ, Ward ES. Using multifocal plane microscopy to reveal novel trafficking processes in the recycling pathway. JCell Sci. 2013;126(Pt 5):1176-1188.

20. Ward ES, Martinez C, Vaccaro C, Zhou J, Tang Q Ober RJ. From sorting endosomes to exocytosis: association of Rab4 and Rab11 GTPases with the Fc receptor, FcRn, during recycling. Mol Biol Cell. 2005;16(4):2028-2038.

21. Abdiche YN, et al. The neonatal Fc receptor 
( $F c R n)$ binds independently to both sites of the IgG homodimer with identical affinity. MAbs. 2015;7(2):331-343.

22. Clynes R. Protective mechanisms of IVIG. Curr Opin Immunol. 2007;19(6):646-651.

23. Dirks NL, Meibohm B. Population pharmacokinetics of therapeutic monoclonal antibodies. Clin Pharmacokinet. 2010;49(10):633-659.

24. Martins JP, Kennedy PJ, Santos HA, Barrias C, Sarmento B. A comprehensive review of the neonatal $\mathrm{Fc}$ receptor and its application in drug delivery. Pharmacol Ther. 2016;161:22-39.

25. Dall'Acqua WF, et al. Increasing the affinity of a human IgG1 for the neonatal Fc receptor: biological consequences. JImmunol. 2002;169(9):5171-5180.

26. Borrok MJ, et al. pH-dependent binding engineering reveals an FcRn affinity threshold that governs IgG recycling. J Biol Chem. 2015;290(7):4282-4290.

27. Vaccaro C, Bawdon R, Wanjie S, Ober RJ, Ward ES. Divergent activities of an engineered antibody in murine and human systems have implications for therapeutic antibodies. Proc Natl Acad SciU S A. 2006;103(49):18709-18714.

28. Waldmann TA, Terry WD. Familial hypercatabolic hypoproteinemia. A disorder of endogenous catabolism of albumin and immunoglobulin. J Clin Invest. 1990;86(6):2093-2098.

29. Wani MA, et al. Familial hypercatabolic hypoproteinemia caused by deficiency of the neonatal Fc receptor, FcRn, due to a mutant beta2microglobulin gene. Proc Natl Acad Sci U S A. 2006;103(13):5084-5089.

30. Derksen RH, Schuurman HJ, Gmelig Meyling FH, Struyvenberg A, Kater L. Rebound and overshoot after plasma exchange in humans. J Lab Clin Med. 1984;104(1):35-43.

31. Neuber $\mathrm{T}$, et al. Characterization and screening of IgG binding to the neonatal Fc receptor. MAbs. 2014;6(4):928-942.

32. West AP, Bjorkman PJ. Crystal structure and immunoglobulin $G$ binding properties of the human major histocompatibility complex-related Fc receptor. Biochemistry. 2000;39(32):9698-9708.

33. Weflen AW, et al. Multivalent immune complexes divert FcRn to lysosomes by exclusion from recycling sorting tubules. Mol Biol Cell.
2013;24(15):2398-2405.

34. Baker K, et al. Neonatal Fc receptor expression in dendritic cells mediates protective immunity against colorectal cancer. Immunity. 2013;39(6):1095-1107.

35. Liu JF, et al. Comparing the autoantibody levels and clinical efficacy of double filtration plasmapheresis, immunoadsorption, and intravenous immunoglobulin for the treatment of late-onset myasthenia gravis. Ther Apher Dial. 2010;14(2):153-160.

36. Schmidt E, et al. Protein A immunoadsorption: a novel and effective adjuvant treatment of severe pemphigus. Br J Dermatol. 2003;148(6):1222-1229.

37. Snyder HW, Bertram JH, Channel M, Ernst NR, Balint JP, Jones FR. Reduction in platelet-binding immunoglobulins and improvement in platelet counts in patients with HIV-associated idiopathic thrombocytopenia purpura (ITP) following extracorporeal immunoadsorption of plasma over staphylococcal protein A-silica. ArtifOrgans. 1989;13(1):71-77.

38. Grevys A, et al. Fc Engineering of human IgG1 for altered binding to the neonatal Fc receptor affects Fc effector functions. JImmunol. 2015;194(11):5497-5508.

39. Furst DE. Serum immunoglobulins and risk of infection: how low can you go? Semin Arthritis Rheum. 2009;39(1):18-29.

40. Jacob S, Rajabally YA. Current proposed mechanisms of action of intravenous immunoglobulins in inflammatory neuropathies. Curr Neuropharmacol. 2009;7(4):337-342.

41. Ogbogu P, Hostoffer RW. Analysis of headaches associated with gammaglobulin infusion. JAllergy Clin Immunol. 2004;113(Suppl_2):S122.

42. Finkel AG, Howard JF, Mann JD. Successful treatment of headache related to intravenous immunoglobulin with antimigraine medications. Headache. 1998;38(4):317-321.

43. Kobrynski L. Subcutaneous immunoglobulin therapy: a new option for patients with primary immunodeficiency diseases. Biologics. 2012;6:277-287.

44. Stiehm ER. Adverse effects of human immunoglobulin therapy. Transfus Med Rev.
2013;27(3):171-178.

45. Oshima $\mathrm{H}$, et al. Characterization of murine CD70 by molecular cloning and mAb. Int Immunol. 1998;10(4):517-526.

46. Foote J, Winter G. Antibody framework residues affecting the conformation of the hypervariable loops. J Mol Biol. 1992;224(2):487-499.

47. Popov S, Hubbard JG, Kim J, Ober B, Ghetie V, Ward ES. The stoichiometry and affinity of the interaction of murine $\mathrm{Fc}$ fragments with the MHC class I-related receptor, FcRn. Mol Immunol. 1996;33(6):521-530.

48. Firan M, et al. The MHC class I-related receptor, FcRn, plays an essential role in the maternofetal transfer of gamma-globulin in humans. Int Immunol. 2001;13(8):993-1002.

49. Ober RJ, Caves J, Ward ES. Analysis of exponential data using a noniterative technique: application to surface plasmon experiments. Anal Biochem. 2003;312(1):57-65.

50. Zhou J, Mateos F, Ober RJ, Ward ES. Conferring the binding properties of the mouse MHC class I-related receptor, FcRn, onto the human ortholog by sequential rounds of site-directed mutagenesis. JMol Biol. 2005;345(5):1071-1081.

51. Chao J, Ward ES, Ober RJ. A software framework for the analysis of complex microscopy image data. IEEE Trans Inf Technol Biomed. 2010;14(4):1075-1087.

52. van Dongen JJ, et al. EuroFlow antibody panels for standardized $n$-dimensional flow cytometric immunophenotyping of normal, reactive and malignant leukocytes. Leukemia. 2012;26(9):1908-1975.

53. Gough K, et al. Assessment of dose proportionality: report from the statisticians in the Pharmaceutical Industry/Pharmacokinetics UK Joint Working Party. Drug Inf J. 1995;29(3):1039-1048.

54. Deisenhofer J. Crystallographic refinement and atomic models of a human Fc fragment and its complex with fragment B of protein A from Staphylococcus aureus at 2.9- and 2.8-A resolution. Biochemistry. 1981;20(9):2361-2370.

55. Kim JK, Firan M, Radu CG, Kim CH, Ghetie V, Ward ES. Mapping the site on human IgG for binding of the MHC class I-related receptor, FcRn. Eur J Immunol. 1999;29(9):2819-2825. 